\title{
Mass Calibration of Optically Selected DES Clusters Using a Measurement of CMB-cluster Lensing with SPTpol Data
}

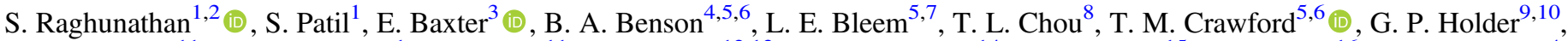
T. McClintock ${ }^{11}$, C. L. Reichardt ${ }^{1}$ (i), E. Rozo ${ }^{11}$, T. N. Varga ${ }^{12,13}$, T. M. C. Abbott ${ }^{14}$, P. A. R. Ade $^{15}$ (1) , S. Allam ${ }^{16}$, A. J. Anderson ${ }^{4}$, J. Annis ${ }^{16}$, , J. E. Austermann ${ }^{17}$, S. Avila ${ }^{18}$, J. A. Beall ${ }^{17}$, K. Bechtol ${ }^{19}$, A. N. Bender ${ }^{5,7}$, G. Bernstein ${ }^{3}$, E. Bertin ${ }^{20,21}$, F. Bianchini ${ }^{1}$ (1), D. Brooks ${ }^{22}$, D. L. Burke ${ }^{23,24}$, J. E. Carlstrom ${ }^{5,6,7,25,26}$, J. Carretero ${ }^{27}$, C. L. Chang ${ }^{5,6,7}$, H. C. Chiang ${ }^{28}$, H-M. Cho ${ }^{29}$, R. Citron ${ }^{5}$, A. T. Crites ${ }^{5,6,30}$, C. E. Cunha ${ }^{23}$, L. N. da Costa $^{31,32}$, C. Davis ${ }^{23}$, S. Desai ${ }^{33}$, H. T. Diehl $^{16}$,

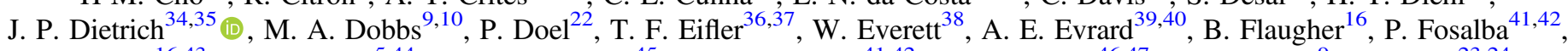
J. Frieman $^{16,43}$, J. Gallicchio ${ }^{5,44}$, J. García-Bellido ${ }^{45}$ (i), E. Gaztanaga ${ }^{41,42}$, E. M. George ${ }^{46,47}$ (i), A. Gilbert ${ }^{9}$, D. Gruen ${ }^{23,24}$ (i),

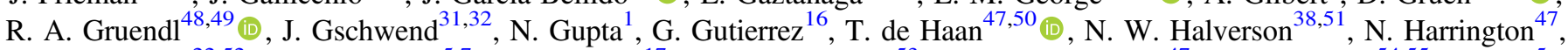

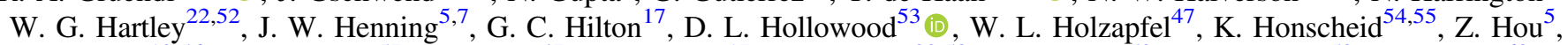
B. Hoyle ${ }^{12,56}$, J. D. Hrubes ${ }^{57}$, N. Huang ${ }^{47}$, J. Hubmayr ${ }^{17}$, K. D. Irwin ${ }^{29,58}$, D. J. James ${ }^{59}$ (1), T. Jeltema ${ }^{53}$, A. G. Kim ${ }^{60}$,

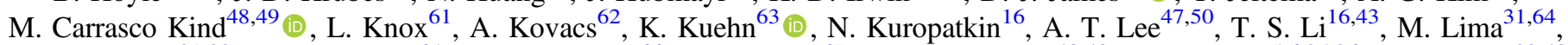
M. A. G. Maia' ${ }^{31,32}$, J. L. Marshall ${ }^{65}$, J. J. McMahon ${ }^{66}$, P. Melchior ${ }^{67}$ (1) , F. Menanteau ${ }^{48,49}$, S. S. Meyer ${ }^{5,6,25,26}$, C. J. Miller ${ }^{39,40}$, R. Miquel ${ }^{27,68}$ (1) L. Mocanu ${ }^{5,6}$, J. Montgomery ${ }^{9}$, A. Nadolski ${ }^{69,70}$, T. Natoli ${ }^{5,25,71}$, J. P. Nibarger ${ }^{17}$, V. Novosad ${ }^{72}$, S. Padin $^{5,6,30}$, A. A. Plazas ${ }^{37}$, C. Pryke ${ }^{73}$, D. Rapetti ${ }^{74,75}$ (1) A. K. Romer ${ }^{76}$ (1) A. Carnero Rosell ${ }^{31,32}$, J. E. Ruhl7 ${ }^{77}$, B. R. Saliwanchik ${ }^{28}$ (D), E. Sanchez ${ }^{78}$, J. T. Sayre ${ }^{38,51}$, V. Scarpine ${ }^{16}$, K. K. Schaffer ${ }^{5,26,79}$, M. Schubnell ${ }^{40}$, S. Serrano ${ }^{41,42}$, I. Sevilla-Noarbe ${ }^{78}$, G. Smecher ${ }^{9,80}$, R. C. Smith ${ }^{14}$, M. Soares-Santos ${ }^{81}$ (1) F. Sobreira ${ }^{31,82}$ (1) A. A. Stark ${ }^{83}$, K. T. Story ${ }^{58,84}$, E. Suchyta ${ }^{85}$, M. E. C. Swanson ${ }^{49}$, G. Tarle $^{40}$ (i), D. Thomas ${ }^{18}$, C. Tucker $^{15}$, K. Vanderlinde ${ }^{71,86}$, J. De Vicente ${ }^{78}$, J. D. Vieira ${ }^{69,70}$, G. Wang ${ }^{7}$, N. Whitehorn ${ }^{2}$, W. L. K. Wu ${ }^{5}$ (1), and Y. Zhang ${ }^{16}$ (1)

${ }^{1}$ School of Physics, University of Melbourne, Parkville, VIC 3010, Australia; srinivasan.raghunathan @unimelb.edu.au ${ }^{2}$ Department of Physics and Astronomy, University of California, Los Angeles, CA 90095, USA

${ }^{3}$ Department of Physics and Astronomy, University of Pennsylvania, Philadelphia, PA 19104, USA ${ }^{4}$ Fermi National Accelerator Laboratory, MS209, P.O. Box 500, Batavia, IL 60510, USA

${ }^{5}$ Kavli Institute for Cosmological Physics, University of Chicago, 5640 South Ellis Avenue, Chicago, IL 60637, USA

${ }^{6}$ Department of Astronomy and Astrophysics, University of Chicago, 5640 South Ellis Avenue, Chicago, IL 60637, USA

${ }^{7}$ High Energy Physics Division, Argonne National Laboratory, 9700 S. Cass Avenue, Argonne, IL 60439, USA

8 Department of Physics, University of Chicago, Chicago, IL 60637, USA

${ }^{9}$ Department of Physics, McGill University, 3600 Rue University, Montreal, Quebec H3A 2T8, Canada

${ }^{10}$ Canadian Institute for Advanced Research, CIFAR Program in Cosmology and Gravity, Toronto, ON, M5G 1Z8, Canada

${ }^{11}$ Department of Physics, University of Arizona, Tucson, AZ 85721, USA

${ }^{12}$ Max Planck Institute for Extraterrestrial Physics, Giessenbachstrasse, D-85748 Garching, Germany

${ }^{13}$ Universitäts-Sternwarte, Fakultät für Physik, LudwigMaximilians Universität München, Scheinerstr. 1, D-81679 München, Germany

${ }^{14}$ Cerro Tololo Inter-American Observatory, National Optical Astronomy Observatory, Casilla 603, La Serena, Chile ${ }^{15}$ Cardiff University, Cardiff CF10 3XQ, UK

${ }^{16}$ Fermi National Accelerator Laboratory, P.O. Box 500, Batavia, IL 60510, USA

${ }^{17}$ NIST Quantum Devices Group, 325 Broadway Mailcode 817.03, Boulder, CO 80305, USA

${ }^{18}$ Institute of Cosmology \& Gravitation, University of Portsmouth, Portsmouth, PO1 3FX, UK

${ }^{19}$ LSST, 933 North Cherry Avenue, Tucson, AZ 85721, USA

${ }^{20}$ CNRS, UMR 7095, Institut d'Astrophysique de Paris, F-75014, Paris, France

${ }^{21}$ Sorbonne Universités, UPMC Univ Paris 06, UMR 7095, Institut d'Astrophysique de Paris, F-75014, Paris, France

22 Department of Physics \& Astronomy, University College London, Gower Street, London, WC1E 6BT, UK

${ }^{23}$ Kavli Institute for Particle Astrophysics \& Cosmology, P.O. Box 2450, Stanford University, Stanford, CA 94305, USA

${ }^{24}$ SLAC National Accelerator Laboratory, Menlo Park, CA 94025, USA

${ }_{25}^{25}$ Department of Physics, University of Chicago, 5640 South Ellis Avenue, Chicago, IL 60637, USA

${ }^{26}$ Enrico Fermi Institute, University of Chicago, 5640 South Ellis Avenue, Chicago, IL 60637, USA

${ }^{27}$ Institut de Física d'Altes Energies (IFAE), The Barcelona Institute of Science and Technology, Campus UAB, E-08193 Bellaterra (Barcelona), Spain

${ }^{28}$ School of Mathematics, Statistics \& Computer Science, University of KwaZulu-Natal, Durban, South Africa

${ }^{29}$ SLAC National Accelerator Laboratory, 2575 Sand Hill Road, Menlo Park, CA 94025, USA

${ }^{30}$ California Institute of Technology, MS 249-17, 1216 E. California Boulevard, Pasadena, CA 91125, USA

${ }^{31}$ Laboratório Interinstitucional de e-Astronomia-LIneA, Rua Gal. José Cristino 77, Rio de Janeiro, RJ-20921-400, Brazil

${ }^{32}$ Observatório Nacional, Rua Gal. José Cristino 77, Rio de Janeiro, RJ-20921-400, Brazil

${ }^{33}$ Department of Physics, IIT Hyderabad, Kandi, Telangana 502285, India

${ }^{34}$ Faculty of Physics, Ludwig-Maximilians-Universität, Scheinerstr. 1, D-81679 Munich, Germany

${ }^{35}$ Excellence Cluster Universe, Boltzmannstr. 2, D-85748 Garching, Germany

${ }^{36}$ Department of Astronomy/Steward Observatory, 933 North Cherry Avenue, Tucson, AZ 85721-0065, USA

37 Jet Propulsion Laboratory, California Institute of Technology, 4800 Oak Grove Drive, Pasadena, CA 91109, USA

38 Department of Astrophysical and Planetary Sciences, University of Colorado, Boulder, CO 80309, USA

${ }_{40}$ Department of Astronomy, University of Michigan, Ann Arbor, MI 48109, USA

40 Department of Physics, University of Michigan, Ann Arbor, MI 48109, USA

${ }^{41}$ Institut d'Estudis Espacials de Catalunya (IEEC), E-08193 Barcelona, Spain

${ }^{42}$ Institute of Space Sciences (ICE, CSIC), Campus UAB, Carrer de Can Magrans, s/n, E-08193 Barcelona, Spain

Kavli Institute for Cosmological Physics, University of Chicago, Chicago, IL 60637, USA

${ }^{44}$ Harvey Mudd College, 301 Platt Boulevard, Claremont, CA 91711, USA

${ }^{45}$ Instituto de Fisica Teorica UAM/CSIC, Universidad Autonoma de Madrid, E-28049 Madrid, Spain

${ }^{46}$ European Southern Observatory, Karl-Schwarzschild-Str. 2, D-85748 Garching bei München, Germany 


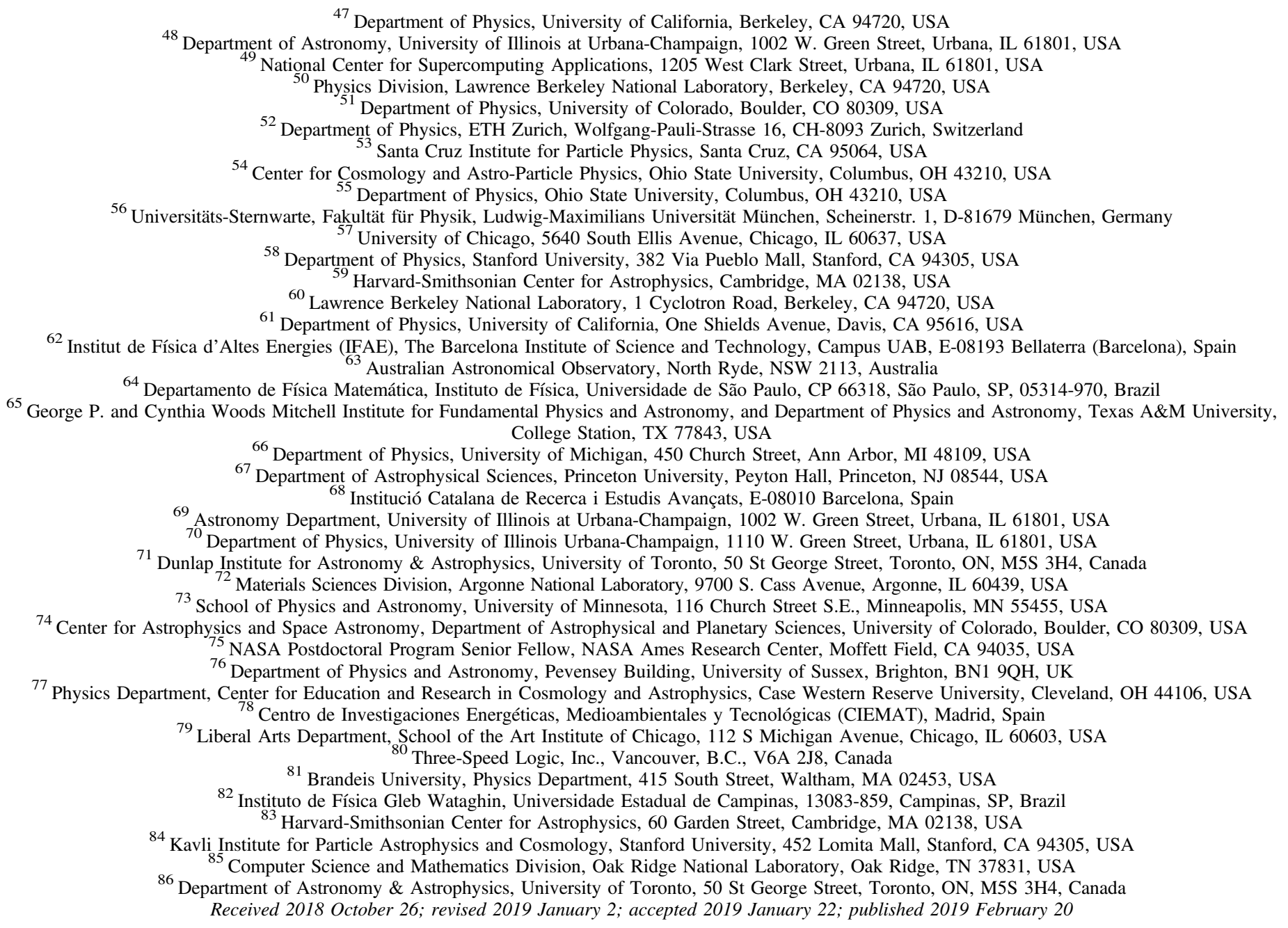

\begin{abstract}
We use cosmic microwave background (CMB) temperature maps from the $500 \mathrm{deg}^{2} \mathrm{SPTpol}$ survey to measure the stacked lensing convergence of galaxy clusters from the Dark Energy Survey (DES) Year-3 redMaPPer (RM) cluster catalog. The lensing signal is extracted through a modified quadratic estimator designed to be unbiased by the thermal Sunyaev-Zel'dovich (tSZ) effect. The modified estimator uses a tSZ-free map, constructed from the SPTpol 95 and $150 \mathrm{GHz}$ data sets, to estimate the background CMB gradient. For lensing reconstruction, we employ two versions of the RM catalog: a flux-limited sample containing 4003 clusters and a volume-limited sample with 1741 clusters. We detect lensing at a significance of $8.7 \sigma(6.7 \sigma)$ with the flux (volume)-limited sample. By modeling the reconstructed convergence using the Navarro-Frenk-White profile, we find the average lensing masses to be $M_{200 \mathrm{~m}}=\left(1.62_{-0.25}^{+0.32}\right.$ [stat.] \pm 0.04 [sys.] $)$ and $\left(1.28_{-0.18}^{+0.14}\right.$ [stat.] \pm 0.03 [sys.] $) \times 10^{14} M_{\odot}$ for the volume- and flux-limited samples, respectively. The systematic error budget is much smaller than the statistical uncertainty and is dominated by the uncertainties in the RM cluster centroids. We use the volume-limited sample to calibrate the normalization of the mass-richness scaling relation, and find a result consistent with the galaxy weak-lensing measurements from DES.
\end{abstract}

Key words: cosmic background radiation - galaxies: clusters: general - gravitational lensing: weak

\section{Introduction}

The abundance of galaxy clusters as a function of mass and redshift is highly sensitive to the the details of structure growth and the geometry of the universe (e.g., Allen et al. 2011). Past cluster surveys have yielded competitive constraints on a number of open questions in cosmology today, most notably on the sum of the neutrino masses and the drivers for cosmic acceleration (Mantz et al. 2008, 2015; Vikhlinin et al. 2009b; Rozo et al. 2010; Hasselfield et al. 2013; de Haan et al. 2016;
Planck Collaboration et al. 2016a; Salvati et al. 2018). Future surveys (LSST Science Collaboration et al. 2009; Merloni et al. 2012; Benson et al. 2014; CMB-S4 Collaboration et al. 2016; Henderson et al. 2016; The Simons Observatory Collaboration et al. 2018) will find tens to hundreds of thousands of galaxy clusters, with the potential for significantly better cosmological constraints. Achieving this improvement, however, will also require a calibration between cluster mass with observable quantities such as X-ray luminosity, the Sunyaev-Zel'dovich 
(SZ) effect, or optical richness (Sunyaev \& Zel'dovich 1972; Sunyaev \& Zeldovich 1980; Vikhlinin et al. 2009a; Rozo et al. 2010; Applegate et al. 2014; von der Linden et al. 2014).

Gravitational lensing is one of the most promising techniques to estimate galaxy cluster masses. Gravitational lensing has the significant advantage that it directly probes the total matter distribution in a galaxy cluster, without depending on complex baryonic physics. Optical weak-lensing measurements have demonstrated accurate mass estimates that have been used in recent cluster cosmological analyses (Rozo et al. 2013; von der Linden et al. 2014). While galaxies may be the most well-known lensing source (Hoekstra et al. 2013), any background light source can be used. The cosmic microwave background (CMB) is an effective alternative due to its extremely well-measured statistical properties and known high redshift $(z \sim 1100)$. CMB-cluster lensing is particularly powerful for high-redshift clusters, for which it is more difficult to observe background galaxies with sufficient signalto-noise $(\mathrm{S} / \mathrm{N})$. Consequently, it is one of the most promising methods for future CMB surveys, including CMB-S4, which are expected to return thousands of high redshift $(z>1)$ clusters (CMB-S4 Collaboration et al. 2016). For low-redshift clusters, CMB lensing is complementary to galaxy weaklensing measurements, as the systematics associated with the two measurements are different. However, the CMB-cluster lensing signal is small. We estimate the lensing $\mathrm{S} / \mathrm{N}$ to be $\sim 0.5$ for a cluster with $M \sim 10^{14} M_{\odot}$, even for a futuristic experiment like CMB-S4. So we are limited to measuring the average mass of a set of clusters.

Several estimators have been proposed to extract the CMBcluster lensing signal using CMB temperature and polarization maps (Seljak \& Zaldarriaga 2000; Dodelson 2004; Holder \& Kosowsky 2004; Maturi et al. 2005; Lewis \& Challinor 2006; $\mathrm{Hu}$ et al. 2007; Yoo \& Zaldarriaga 2008; Yoo et al. 2010; Melin \& Bartlett 2015; Horowitz et al. 2017). Measurements have now been performed by a number of experiments using CMB temperature data. Baxter et al. (2015) detected CMBcluster lensing at 3.1 $\sigma$ using South Pole Telescope (SPT) SZ survey data for a sample 513 SPT-selected clusters. Additional detections of CMB-cluster lensing have been made using ACTPol (Madhavacheril et al. 2015) and Planck data (Planck Collaboration et al. 2016a; Raghunathan et al. 2018). CMBcluster lensing has also been used to calibrate the mass-richness $(M-\lambda)$ relation of the redMaPPer $(\mathrm{RM})$ algorithm using both Planck data at the locations of clusters in Sloan Digital Sky Survey (SDSS, Geach \& Peacock 2017) data, and SPT-SZ data at the locations of clusters in Dark Energy Survey (DES) Year-1 (Baxter et al. 2018, hereafter B18) data.

These initial measurements have estimated the lensing signal from $\mathrm{CMB}$ temperature data. Lensing measurements using temperature data are susceptible to bias from foreground signals-in particular the thermal SZ (tSZ) signal from the cluster itself. The bias due to the tSZ effect can be mitigated by using tSZ-free maps for lensing measurements (Baxter et al. 2015) or by including additional filtering when estimating the background gradient with a lensing quadratic estimator $(\mathrm{QE}, \mathrm{B} 18)$. Both of these methods reduce the lensing $\mathrm{S} / \mathrm{N}$. We follow a different strategy here by reworking the standard QE to use a tSZ-free gradient map from the SPTpol survey for a tSZ-bias free lensing reconstruction. While this paper was in the production stage, Madhavacheril \& Hill (2018) published a similar method using simulated data sets where they also demonstrated that the tSZ-free gradient QEs can robustly reconstruct $\mathrm{CMB}$ lensing using temperature data alone.

In the current work, we apply the modified QE to SPTpol $\mathrm{CMB}$ temperature maps and reconstruct the lensing signal at the location of galaxy clusters from the DES Year-3 RM catalog. We employ two samples of the RM catalog and obtain lensing detection significances of $8.7 \sigma$ with 4003 clusters from the flux-limited sample and $6.7 \sigma$ for a smaller volume-limited sample containing 1741 clusters. We use the lensing measurements from the volume-limited sample to calibrate the $M-\lambda$ relation of the RM cluster sample at the $18 \%$ level. We validate our results against several sources of systematic errors and note that the uncertainty in the knowledge of the cluster miscentering introduces a $\sim 3 \%$ error in our lensing measurements, which is subdominant compared to the statistical error.

The paper is organized as follows. In Section 2 we describe the SPTpol CMB temperature map and the DES RM cluster catalog. This is followed by a description of the lensing estimator, simulations used to validate the pipeline, cluster convergence profiles, cutout extraction, and the modeling in Section 3. Pipeline and data validation along with the estimates of the systematic error budgets are summarized in Section 4 . We present our lensing measurements and compare them to the literature in Section 5. The conclusion is in Section 6.

Throughout this work, we use the best-fit $\Lambda \mathrm{CDM}$ cosmology obtained from the chain that combines Planck 2015 data with external data sets TT, TE, EE+lowP+lensing+ext (Planck Collaboration et al. 2016b). We define all halo quantities with respect to the radius $R_{200 \mathrm{~m}}$, defined as the region within which the average mass density is 200 times the mean density of the universe at the halo redshift. For parameter constraints, we report the median values and $1 \sigma$ uncertainties from the 16 th and 84th percentiles.

\section{Data}

We describe the CMB data sets from the SPTpol survey in Section 2.1. This is followed by a brief description of the DES experiment and the selection of the cluster catalog used in this work in Section 2.2.

\subsection{SPTpol $500 \mathrm{deg}^{2}$ Survey}

SPTpol is the second camera installed on the $10 \mathrm{~m}$ SPT (Padin et al. 2008; Carlstrom et al. 2011) located at the Amundsen-Scott South Pole station. The SPTpol focal plane consists of 1536 polarization-sensitive transition edge sensor bolometers $(360$ at $95 \mathrm{GHz}$ and 1176 at $150 \mathrm{GHz}$; Austermann et al. 2012). The SPTpol $500 \mathrm{deg}^{2}$ survey spans 15 degrees of decl., from $-65^{\circ}$ to $-50^{\circ}$, and 4 hours of R.A., from 22 to $2 \mathrm{hr}$. In this work, we use CMB temperature maps from observations between 2013 April and 2016 September in frequency bands centered at approximately 95 and $150 \mathrm{GHz}$. The telescope beam and pointing solutions were characterized using Venus and bright point sources in the SPTpol survey region. The final telescope beam along with the pointing jitter roughly corresponds to a $\theta_{\mathrm{FWHM}}=1 ! 22(1 ! 7)$ Gaussian for the 150 (95) $\mathrm{GHz}$ data set.

We briefly summarize the procedure we use to reduce raw CMB data to maps and refer the reader to Henning et al. (2018) for further details. The raw data are composed of digitized timeordered data (TOD) for each detector that are converted into $\mathrm{CMB}$ temperature units. We bin the TOD into two different maps using 
a flat-sky approximation in the Sanson-Flamsteed projection (Calabretta \& Greisen 2002; Schaffer et al. 2011). To construct the first map, in which we aim to reconstruct the small-scale lensing signal, we remove large-scale modes $\ell \leqslant 300$, bandpass filter the TOD in the range of approximately $300 \leqslant \ell_{x} \leqslant 20,000$, and bin them into 0.5 square pixels. For the second map, intended for estimation of the large-scale CMB gradient, we apply minimal TOD filtering by only removing modes below $\ell_{x} \leqslant 30$, and bin them into $3^{\prime}$ square pixels. While we only use the data from the $150 \mathrm{GHz}$ channel for the first map, the latter is a tSZ signal cleaned map produced by linearly combining the 95 and $150 \mathrm{GHz}$ channels. We use this tSZ-free map to reconstruct the background gradient of the CMB at the cluster locations. As we will see later in Section 3.1, the gradient estimation using the tSZ-free map helps in removing the tSZ-induced lensing bias. The minimal filtering on this map allows us to recover large-scale modes, which indeed helps in a better estimation of the background gradient. The 0.5 resolution $150 \mathrm{GHz}$ map has a white noise level of $\Delta_{T}=6 \mu \mathrm{K}^{\prime}$, estimated using a jackknife approach. The lowresolution tSZ-free combination is noisier with $\Delta_{T} \sim 17 \mu \mathrm{K}^{\prime}$.

\subsection{DES and the redMaPPer Catalog}

The DES is a $\sim 5000 \mathrm{deg}^{2}$, optical to near-infrared survey conducted using the Dark Energy Camera (Flaugher et al. 2015) mounted on the $4 \mathrm{~m}$ Victor Blanco telescope at Cerro Tololo Observatory in Chile, and has recently begun its 6th year of observations. For this analysis, we use the cluster catalog obtained from the first 3 years of DES observations, which almost covers the SPTpol $500 \mathrm{deg}^{2}$ survey.

The cluster catalog was derived using the RM algorithm (Rykoff et al. 2014). RM is an optical cluster-finding algorithm which detects candidates by identifying over-densities of luminous red galaxies with luminosity greater than $20 \%$ of $L_{*}$. It is based on our understanding that galaxy clusters are agglomerations of galaxies containing old and subsequently red stars. The algorithm iteratively assigns membership and centering probabilities for each red galaxy identified as belonging to a cluster candidate. A weighted sum of the membership probabilities, richness $\lambda$, is assigned to each candidate. The center comes from the galaxy with the highest centering probability. The DES RM catalog contains two samples: a flux-limited sample and a volume-limited sample. The flux-limited sample has more high-redshift clusters detected from deep fields in the survey. On the other hand, the volume-limited sample is independent of survey depth, complete above a luminosity threshold (McClintock et al. 2019, hereafter M19), and normally preferred for cosmological analysis. See Rykoff et al. (2016) for more information on the application of RM to the DES survey data.

The RM cluster catalog version employed in this analysis is y3_gold:v6.4.22. The Year-3 gold catalog is based on the previous catalog from the Year-1 data (Drlica-Wagner et al. 2018), with some updates described in Morganson et al. (2018). The catalog contains 54,112 clusters above richness $\lambda \geqslant 20$ in the flux-limited sample and 21,094 clusters in the volume-limited sample. Of these, 5828 (2428) clusters from the flux(volume)-limited sample lie within the SPTpol $500 \mathrm{deg}^{2}$ survey in the redshift range $0.1 \leqslant z \leqslant 0.95$ (0.90). We additionally remove clusters near the survey edges by removing the cutouts (see Section 3.4) with more than 5\% masked pixels or within $10^{\prime}$ distance from any bright $(\geqslant 6 \mathrm{mJy}$ at $150 \mathrm{GHz})$ point sources detected in the SPTpol temperature map. These cuts leave 4003 (1741) clusters with $\lambda \geqslant 20$ from the flux (volume)-limited sample with a median redshift of $\tilde{z}=0.77$ $(0.48)$. The error in the cluster photo- $z$ estimates are small with $\hat{\sigma}_{z}=0.01(1+z)$ (Rozo et al. 2016).

\section{Methods}

We now turn to the method for measuring the cluster lensing signal. First, we describe the modified QE, which uses a tSZfree gradient map to eliminate the tSZ-induced bias in Section 3.1. Next, we present the lensing pipeline starting with the simulations used in the analysis in Section 3.2, calculation of the cluster convergence profiles in Section 3.3, cluster cutouts extraction in Section 3.4, the weighting scheme applied to obtain the stacked convergence in Section 3.5, and modeling in Section 3.6.

\subsection{Quadratic Estimator}

We use a quadratic lensing estimator ( $\mathrm{Hu}$ et al. 2007) to extract the cluster lensing signal. Specifically, we obtain the convergence $\kappa$ which is related to the underlying lensing potential $\phi$ as $2 \kappa=-\nabla^{2} \phi$. The QE uses two maps to reconstruct the lensing convergence: one map of the $\mathrm{CMB}$ gradient on large scales, and one map of the CMB temperature fluctuations on small scales. In the absence of lensing, the two maps would be uncorrelated. The convergence reconstructed from the two maps will be ( $\mathrm{Hu}$ et al. 2007)

$$
\hat{\kappa}_{\ell}=-A_{\ell} \int d^{2} \hat{\boldsymbol{n}} e^{-i \hat{\boldsymbol{n}} \cdot \ell} \operatorname{Re}\left\{\nabla \cdot\left[G(\hat{\boldsymbol{n}}) L^{*}(\hat{\boldsymbol{n}})\right]\right\},
$$

where $G$ is the temperature gradient map and $L$ is the temperature fluctuation map, both optimally filtered to maximize the lensing $\mathrm{S} / \mathrm{N}$. The two maps and the optimal weights are described in the next section. The normalization factor $A_{\ell}$ can be calculated following Equation (18) of $\mathrm{Hu}$ et al. (2007). Since the desired input to the QE is the gradient of the unlensed $\mathrm{CMB}$, the gradient map $G$ is low-pass filtered (LPF) at $\ell_{G}(\mathrm{Hu}$ et al. 2007) to avoid multipoles where the cluster lensing or foregrounds begin to enter. The LPF negligibly degrades the lensing $\mathrm{S} / \mathrm{N}$, since most of the gradient information is at large scales (see Figure 1 of $\mathrm{Hu}$ et al. 2007).

When, as in this work, temperature maps are used in both legs of the QE, lensing is not the only process that introduces correlations between the maps $G$ and $L$. Undesired correlations are also sourced by clusters' own SZ signals; these correlations lead to severe contamination of the lensing reconstruction. An obvious way to reduce the tSZ bias would be to generate a tSZfree map from a linear combination of single-frequency maps; this has been done in previous analyses (Baxter et al. 2015). However, this linear combination can substantially increase the map noise and degrade the lensing S/N. For instance, the tSZfree map used by Baxter et al. (2015) had a noise level approximately three times higher than the SPT-SZ $150 \mathrm{GHz}$ map alone. Modeling the tSZ signal is possible in principle as an alternative, but we do not yet have an adequate understanding of the intracluster medium to do so reliably.

Modifying the LPF in the gradient map, $\ell_{G}$, is another plausible alternative to reduce but not eliminate this correlation. The lensing bias due to this correlation will be particularly large for massive nearby clusters that span a large angular extent on the sky. While reducing the bias, adopting a lower $\ell_{G}$ 
will reduce the number of modes for the gradient estimation and result in a lower $\mathrm{S} / \mathrm{N}$. Thus, the choice of $\ell_{G}$ is a trade-off between $\mathrm{S} / \mathrm{N}$ and biases due to both the magnification effect considered by $\mathrm{Hu}$ et al. (2007) and from foreground emission. For example, B18, using the SPT-SZ temperature maps $\left(\Delta_{T}=18 \mu \mathrm{K}^{\prime}\right)$, chose $\ell_{G}=1500$ and reported an upper limit of $11 \%$ on the tSZ-induced bias due to clusters in the richness range $\lambda \in[20,40]$.

\subsection{1. $t$ SZ-free Map for Gradient Estimation}

A key point in this analysis is that for QE-based lensing reconstruction, we only need to eliminate tSZ-induced correlations between the maps $G$ and $L$ used in the two legs, which can be done by removing the tSZ signal from either one of the maps. Hence, instead of treating $\ell_{G}$ as a free parameter used to reduce the tSZ bias, we eliminate the bias completely by working with a tSZ-free map, $T^{\mathrm{SZ}-\text { free }}$, for the gradient estimation G. Recently, Madhavacheril \& Hill (2018) also made a successful demonstration of this method independently using simulations. In this analysis, the $T^{\mathrm{SZ}-\text { free }}$ map $G$ is a linear combination of the SPTpol 95 and $150 \mathrm{GHz}$ temperature data. The second map $L$ is the lower-noise SPTpol $150 \mathrm{GHz}$ data, $T^{150}$, alone.

We can now write down expressions for the two maps, $G$ and $L$ :

$$
\begin{gathered}
G_{\ell}=i \ell W_{\ell}^{G} T_{\ell}^{\mathrm{SZ}-\text { free }}, \\
L_{\ell}=W_{\ell}^{L} T_{\ell}^{150} .
\end{gathered}
$$

Here, $W_{\ell}^{G}$ and $W_{\ell}^{L}$ are the optimal linear filters (Hu et al. 2007) to maximize the lensing $\mathrm{S} / \mathrm{N}$ :

$$
\begin{aligned}
W_{\ell}^{G} & = \begin{cases}C_{\ell}^{\text {unl }}\left(C_{\ell}+N_{\ell}^{\mathrm{SZ}-\text { free }}\right)^{-1}, & \ell \leqslant \ell_{G} \\
0, & \text { otherwise }\end{cases} \\
W_{\ell}^{L} & =\left(C_{\ell}+N_{\ell}^{150}\right)^{-1},
\end{aligned}
$$

with $\left(C_{\ell}^{\text {unl }}\right) C_{\ell}$ corresponding to (un)lensed CMB temperature power spectra calculated using the Code for Anisotropies in the Microwave Background (CAMB, ${ }^{87}$ Lewis et al. 2000). $N_{\ell}$ is the noise spectrum for the indicated map, after deconvolving the beam and filter transfer function given in Equation (5). We also add estimates of foreground power such as SZ, CIB, and radio galaxy emission, based on measurements by George et al. (2015), into $N_{\ell}$. As described previously, $\ell_{G}$ is chosen to remove the magnification bias discussed by $\mathrm{Hu}$ et al. (2007) and additionally to suppress power from signals other than the primary unlensed CMB. We set $\ell_{G}=2000$ for clusters with richness $\lambda<60$. For the rest, we use $\ell_{G}=1000$, as the convergence signal from these massive clusters can cause a negative bias in the estimate of the background gradient. While this is a sharp change in $\ell_{G}$, we will see later that it causes a negligible effect in our final $\mathrm{S} / \mathrm{N}$.

Although this method essentially eliminates the tSZ bias, creating a tSZ-free map can enhance other foregrounds (relative to the $\mathrm{CMB}$ ) along with the noise. We look into possible biases from other foregrounds in Section 4.2.1 using the simulations from Sehgal et al. (2010, hereafter S10).

\footnotetext{
${ }^{87}$ https://camb.info/
}

\subsection{Simulations of the Microwave Sky}

In this section, we describe the simulations used for the pipeline validation. We calculate the large-scale structure lensed CMB power spectra for the fiducial Planck 2015 cosmology (Planck Collaboration et al. 2016b) using CAMB. and create $300^{\prime} \times 300^{\prime}$ Gaussian realizations of the $\mathrm{CMB}$ temperature map with 0 ! 25 pixel resolution. ${ }^{88}$ Given the small angular extent, these simulations are done in the flat-sky approximation. These simulations are then lensed using the simulated galaxy cluster convergence profiles from the next section. Next we apply frequency-dependent foreground realizations (see Section 4). The simulated maps are convolved by the beam functions, and are rebinned to 0.5 pixels to reduce the computational requirements.

For realistic simulations, we must also account for the noise and the filtering applied to the real data. We add instrumental noise realizations corresponding to SPTpol maps (see Section 2.1). We follow B18 and other SPT works and approximate the map filtering using a function of the form:

$$
F_{\bar{\ell}}=e^{-\left(\ell_{1} /|\bar{\ell}|\right)^{6}} e^{-\left(\ell_{2} / \ell_{x}\right)^{6}} e^{-\left(\ell_{x} / \ell_{3}\right)^{6}} .
$$

We validate the robustness of this approximation in Section 4.2.3. For the small-scale lensing map, we set $\ell_{1}=300, \ell_{2}=300$, and $\ell_{3}=20,000$. For the gradient map, we set $\ell_{1}=0$ (as the gradient map does not have an isotropic filter), $\ell_{2}=30$, and $\ell_{3}=3000$.

\subsection{Cluster Convergence Profile}

Now we summarize the method to model the convergence signal at cluster locations. The total convergence $\kappa(M, z)$ profile for a galaxy cluster includes contributions from its own matter over-density (the 1-halo term), as well as from correlated structures along the line of sight (the 2-halo term; Seljak 2000; Cooray \& Sheth 2002). For the 1-halo term, $\kappa^{1 h}(M, z)$, we use the Navarro-Frenk-White (NFW, Navarro et al. 1996) profile in Equation (6) to model the underlying dark matter (DM) density profile of the DES RM galaxy clusters,

$$
\rho(r)=\frac{\rho_{0}}{\left(\frac{r}{R_{\mathrm{s}}}\right)\left(1+\frac{r}{R_{\mathrm{s}}}\right)^{2}},
$$

where $R_{\mathrm{S}}$ is the scale radius and $\rho_{0}$ is the central cluster density. In Section 4.2.2 we quantify the robustness of the inferred masses to this assumption by instead using the Einasto DM profile (Einasto \& Haud 1989). We use the photometric redshift measurements in the DES RM cluster catalog and use the Duffy et al. (2008) halo concentration formula to obtain the concentration parameter $c_{200}(M, z)=R_{200} / R_{\mathrm{s}}$. The convergence profile $\kappa^{1 h}(\theta)$ at a radial distance $\theta$ for a spherically symmetric lens like NFW is the ratio of the surface mass density of the cluster and the critical surface density of the universe at the cluster redshift $\Sigma(\theta) / \Sigma$ (crit). To get the NFW convergence profile, we adopt the closed-form expression given by Equation (2.8) of Bartelmann (1996).

When evaluating the pipeline using mock cluster data sets, we leave out the 2-halo term. For the real data, we also consider the lensing arising from structures surrounding the

\footnotetext{
${ }^{88}$ We have confirmed that the results are unchanged when going to smaller initial pixels.
} 
cluster. We model the 2-halo term contribution, $\kappa^{2 h}(M, z)$, to the total lensing convergence using Equation (13) of Oguri \& Hamana (2011). The bias $b_{h}(M, z)$ of a halo with mass $M \equiv M_{200 \mathrm{~m}}$ was calculated adopting the Tinker et al. (2010) formalism. Finally, we correct the cluster convergence profile $\kappa^{1 h}(M, z)$ for the uncertainties in the DES cluster centroids in Section 3.6.

\subsection{Cluster Cutouts}

We now describe the process of extracting cluster cutouts from SPTpol maps. The lensing QE described previously is applied to these cutouts to reconstruct the lensing signal. We extract $300^{\prime} \times 300^{\prime}$ cutouts from the SPTpol temperature (tSZfree and $150 \mathrm{GHz}$ ) maps around each cluster from the DES RM cluster catalog. This corresponds to a roughly $\sim 135 \mathrm{Mpc}$ region around a cluster at $\tilde{z}=0.77$. While the cutout size is much larger than the virial radius of the cluster, we emphasize it is necessary to robustly reconstruct the lensing signal using the background CMB. This is because the amplitude of the lensing signal is proportional to the level of the background gradient, and the CMB has power on scales much larger than the typical cluster size of a few arcminutes. Performing the analysis with smaller cutouts will reduce the $S / N$ of the estimated CMB gradient and affect the final lensing $\mathrm{S} / \mathrm{N}$. After extracting the lensing signal, we limit the modeling and likelihood calculations to a $10^{\prime}$ region around the cluster.

\subsection{Stacked Convergence and the Weighting Scheme}

The lensing $\mathrm{S} / \mathrm{N}$ for a single cluster is much less than unity, and we must stack the lensing signal from several clusters to achieve a reasonable $\mathrm{S} / \mathrm{N}$. Thus the stacked convergence map is simply

$$
\hat{\kappa}=\frac{\sum_{j} w_{j}\left[\hat{\kappa}_{j}-\left\langle\hat{\kappa}_{j}\right\rangle\right]}{\sum_{j} w_{j}}-\hat{\kappa}_{\mathrm{MF}},
$$

where $\hat{\kappa}_{j}$ refers to reconstructed convergence map of cluster $j$ and the weighting scheme $w$ is described as follows. From the stacked map, we remove all modes above the SPTpol $150 \mathrm{GHz}$ beam scale of $\theta_{\mathrm{FWHM}} \sim 1$ ! 2 . We also remove an estimate of the mean field $\hat{\kappa}_{\mathrm{MF}}$ from this stacked convergence map. The mean field arises because of two reasons: The first is because the temperature maps, before being filtered using Equation (2), are apodized using a Hanning window ${ }^{89}$ with a $10^{\prime}$ edge taper to reduce edge effects. The other reason is the presence of inhomogeneous noise in the survey region. We obtain the mean-field bias by stacking the convergence maps reconstructed at 50,000 random locations in the maps.

Weighting scheme: We decompose the weights for each cluster into two components: The first is the inverse-noisevariance weight, $w_{k}$, constructed from the observed standard deviation $\sigma_{\kappa}$ in the reconstructed SPTpol convergence maps in a ring between $10^{\prime}$ and $30^{\prime}$ around the cluster. The noise in convergence is proportional to the noise in the associated gradient map and increases, as expected, when $\ell_{G}$ is reduced. The second ${ }^{90}$ weight comes from the noise in the convergence maps due to the presence of tSZ signal in the second leg of the QE,

\footnotetext{
89 http://mathworld.wolfram.com/HanningFunction.html

90 We note that for the mean field reconstructed from random locations, we only apply the weight $w=1 / \sigma_{\kappa}^{2}$ for stacking.
}

the SPTpol $150 \mathrm{GHz}$ map. While our method completely eliminates the tSZ-induced lensing bias, the presence of the tSZ signal in the second map tends to increase the variance in the convergence maps. The noise is proportional to the tSZ brightness and, as expected, is higher for massive clusters. For example, the lensing signal of a cluster is proportional to its mass $M$, while the tSZ signal scales roughly as $M^{5 / 3}$.

We obtain this second set of weights, $w_{\mathrm{SZ}}$, using simulations. For every cluster in the DES sample, we reconstruct the convergence profile using a simulated tSZ-free gradient map and a $150 \mathrm{GHz}$ map with tSZ signal, assuming an Arnaud profile (Arnaud et al. 2010) with a log-normal scatter of $20 \%$ in the $Y_{\mathrm{SZ}}-M$ relation. We turn off cluster lensing, as the objective here is to only get an estimate of the tSZ-induced noise in the convergence maps. A total of 25 simulations were used to get the noise estimate for each DES cluster. The weights are estimated as $w_{\mathrm{SZ}}=1 / \sigma_{\mathrm{SZ}}^{2}$, where $\sigma_{\mathrm{SZ}}$ is the standard deviation of the "null" convergence map within an angular distance of $10^{\prime}$ from the cluster center. The errors increase with richness and take a power-law form parameterized as $\sigma_{\mathrm{SZ}}(\lambda)=\sigma_{0} \lambda^{\alpha}$ with values $\left(\sigma_{0}, \alpha\right)=(0.0045,1.55)$. The results are unchanged if we derive the weights using the tSZ signal from $\mathrm{S} 10$. The total weight is now

$$
w=\frac{1}{\sigma_{\kappa}^{2}+\sigma_{\mathrm{SZ}}^{2}} .
$$

Introducing $w_{\mathrm{SZ}}$ down-weights the most massive clusters, reducing the contribution of clusters with $\lambda \geqslant 60$ to less than $1 \%$ in the final stacked sample. This is why the change in gradient-map LPF scale to $\ell_{G}=1000$ from the fiducial $\ell_{G}=2000$ for these clusters (see Section 3.1.1) has negligible effects in our final results.

An alternative to this down-weighting is to swap the maps in the two legs of the QE (i.e., the $150 \mathrm{GHz}$ map for the gradient estimation and the tSZ-free map to reconstruct lensing) for clusters with $\sigma_{\mathrm{SZ}}>\sigma_{\kappa}$, which is approximately true for clusters with $\lambda>40$. However, this results in a minimal gain, as the SPTpol tSZ-free map has a higher noise $(\times 3)$ compared to the SPTpol $150 \mathrm{GHz}$ maps. Some other approaches to handle the additional noise from the tSZ signal include (a) rotating the reconstructed lensing map based on the direction of the background $\mathrm{CMB}$ gradient and fitting for the tSZ-noise, and (b) removing a matched-filter estimate of the tSZ signal from the $150 \mathrm{GHz}$ map before passing the map into the QE. We will explore such possibilities in detail in a future work (S. Patil et al. 2018, in preparation).

\subsection{Model Fitting}

We radially bin the stacked convergence map $\hat{\kappa}$ for the likelihood calculation in Equation (9). To obtain the average lensing mass of the DES RM cluster sample, we need to compare this observed, radially binned, stacked convergence profile to convergence models generated using an assumed halo profile. Essentially, we create a convergence model for every cluster using the NFW profile (see Section 3.3) as a function of mass and the cluster redshift $\kappa^{1 h}(M, z)$, add the two halo term $\kappa^{2 h}(M, z)$, filter the model as per the real data, and then stack all the clusters using the weights described in the previous section.

The convergence model $\kappa^{1 h}(M, z)$ must be slightly modified to account for the uncertainties in the RM cluster centroids. Rykoff et al. (2016) compared the centroids of DES RM 
clusters with SZ (Bleem et al. 2015) and X-ray observations and found a fraction, $f_{\text {mis }}=0.22 \pm 0.11$, of the DES clusters to be mis-centered by $\sigma_{\mathrm{R}}$, which is a fraction of the cluster radius $R_{\lambda}=(\lambda / 100)^{0.2} h^{-1} \mathrm{Mpc}$. They further modeled the mis-centering as a Rayleigh distribution with $\sigma_{\mathrm{R}}=c_{\mathrm{mis}} R_{\lambda}$ where $\ln c_{\text {mis }}=-1.13 \pm 0.22$. Mis-centering ought to smear the convergence profiles, and we use the prescription provided in Equation (34) of Oguri \& Takada (2011) to account for the cluster mis-centering. We set $f_{\text {cen }}=1-f_{\text {mis }}=0.78$ and $\sigma_{\mathrm{s}}=\sigma_{\mathrm{R}} / D_{\mathrm{A}}(z)$, where $\sigma_{\mathrm{R}}$ is picked from the Rayleigh distribution (Rykoff et al. 2016) and $D_{\mathrm{A}}(z)$ is the angular diameter distance at the cluster redshift $z$. After the miscentering correction, we filter the model using the approximation to the data filtering in Equation (5) and remove all modes above the SPTpol $150 \mathrm{GHz}$ beam similar to the data. The filtered model of all the individual clusters is weighted (Section 3.5), stacked, and radially binned.

With the model prediction in hand, we can then write down the likelihood of observing the real data as

$$
\begin{aligned}
& -2 \ln \mathcal{L}(\hat{\kappa}(\theta) \mid M) \\
& =\sum_{\theta=0}^{10^{\prime}}[\hat{\kappa}(\theta)-\kappa(\theta)] \hat{C}^{-1}[\hat{\kappa}(\theta)-\kappa(\theta)]^{T},
\end{aligned}
$$

where $\hat{\kappa}(\theta), \kappa(\theta)$ are the azimuthally averaged radial profiles of the stacked data and model convergences, respectively, binned in 10 linearly spaced intervals with $\Delta \theta=1^{\prime}$. To obtain the covariance matrix, we use a jackknife re-sampling technique. We divide the SPTpol $500 \mathrm{deg}^{2}$ region into $N=500$ subfields and estimate the covariance matrix for the radially binned convergence profile as

$$
\hat{C}=\frac{N-1}{N} \sum_{j=1}^{N=500}\left[\hat{\kappa}_{j}(\theta)-\langle\hat{\kappa}(\theta)\rangle\right]\left[\hat{\kappa}_{j}(\theta)-\langle\hat{\kappa}(\theta)\rangle\right]^{T},
$$

where $\hat{\kappa}_{j}(\theta)$ is the azimuthally binned stacked convergence of all the clusters in the $j$ th subfield and $\langle\hat{\kappa}(\theta)\rangle$ is the ensemble average of all 500 subfields. We test this approach by alternatively estimating the covariance matrix using 500 realizations of the random convergence stacks. We do not note any significant differences between the uncertainties estimated using the two approaches. We apply the Hartlap et al. (2007) correction to $\hat{C}^{-1}$ to account for the noise in covariance estimation due to the finite number of jackknife resampling.

\section{Data and Pipeline Validation}

In this section, we describe tests used to investigate the known and unknown systematic effects in the data and to validate the pipeline. We start with the test for unknown systematics through the "curl" null test (Section 4.1). Next we calculate the expected systematic error budget from known sources of systematic uncertainty (Section 4.2).

\section{1. "Curl” Null Test}

We perform a "curl" null test (Hu et al. 2007) at 4003 cluster locations from the DES RM Year-3 flux-limited sample. Specifically, we replace the divergence of the gradient field, $\nabla \cdot\left[G(\hat{\boldsymbol{n}}) L^{*}(\hat{\boldsymbol{n}})\right]$, in Equation (1) with the curl operator. Since the curl of a gradient field is zero, the reconstructed field should

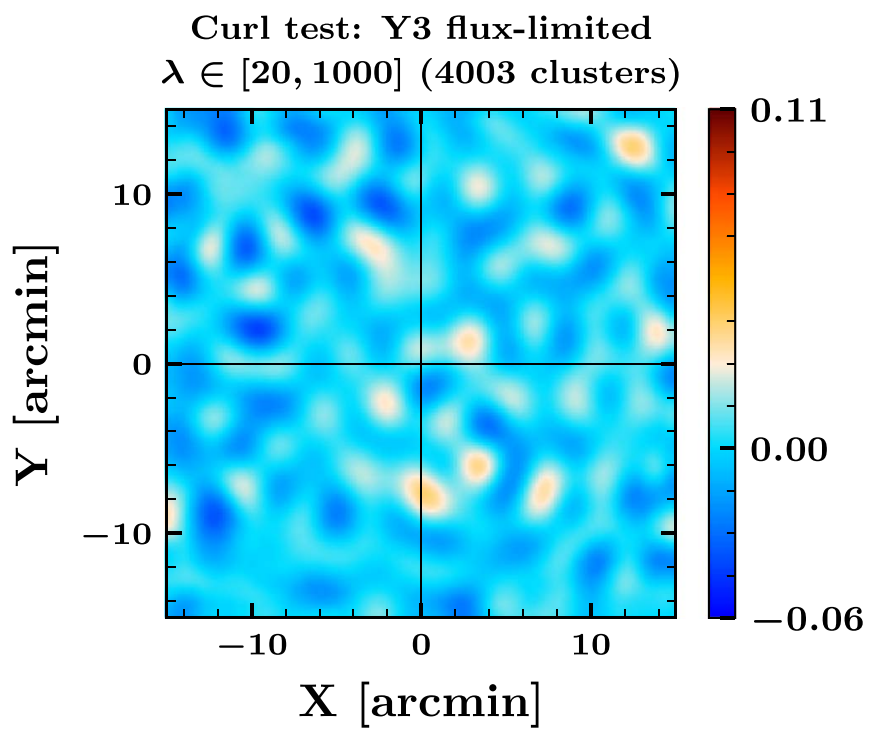

Figure 1. Stacked result of the curl test performed at the cluster locations by replacing the divergence operator in Equation (1) with a curl operator. We obtain a PTE value of 0.26 , consistent with a null result. For the ease of visual comparison, we adopt the same color scale as in Figure 3.

be consistent with zero, unless there is a systematic bias in the data. The result of the curl test is shown in Figure 1. We radially bin the test result similar to the cluster stack as described in Section 3.6 and compare it to a zero signal. The test returns a probability to exceed (PTE) value of 0.26 , consistent with a null signal.

\subsection{Systematic Error Budget}

Now we consider possible sources of systematic error. We estimate the bias due to each cluster's tSZ emission and residual foregrounds, the assumption of an underlying cluster profile, uncertainties in the DES RM mis-centering parameter $f_{\text {mis }}$, approximations to the filter transfer function (Equation (5)), uncertainties in the beam measurements, and the assumption of a background cosmology. Another source of systematic error is the uncertainties in the cluster redshifts, estimated photometrically. However, the impact of photo- $z$ errors was estimated to be negligible by R17, and we ignore them here.

We rely on the $\mathrm{S} 10$ simulations to estimate the level of residual-tSZ/foreground bias in the RM Year-3 flux-limited sample. In all the other cases, we use the data and report the shift in the average lensing mass of the clusters in the RM Year-3 volume-limited sample obtained in Section 5. The combined systematic error budget is presented in Table 1. The systematic error calculated as a quadrature sum of the errors presented in Table 1 is much smaller than the statistical error in the measurements at a level of $0.15 \sigma$. Using a direct sum, the combined error budget is $0.27 \sigma$. The dominant error comes from the uncertainty in the DES RM cluster centroids shifting the mean lensing mass by $2.8 \%$.

\subsubsection{Cluster tSZ Signal and Residual Foregrounds}

In this work, we eliminate the bias due to tSZ signal in the reconstructed lensing maps using SZ-free maps to estimate the background gradient of the CMB. However, projecting just the tSZ signal out tends to modify other frequency-dependent foregrounds, and the resultant map is not an optimal 
Table 1

Systematic Error Budget in the Stacked Mass for DES RM Year-3 Volumelimited Sample

\begin{tabular}{lcc}
\hline \hline \multirow{2}{*}{ Source of Error } & \multicolumn{2}{c}{ Magnitude of Error } \\
\cline { 2 - 3 } & $\%$ & frac. of $\sigma_{\text {stat }}$ \\
\hline Beam uncertainties & $<0.01 \%$ & $\ldots$ \\
Cluster mis-centering & $2.78 \%$ & $0.12 \sigma$ \\
Cosmology & $0.39 \%$ & $0.03 \sigma$ \\
Filtering $\ell_{x}$ & $0.21 \%$ & $0.02 \sigma$ \\
Halo profile & $0.12 \%$ & $0.01 \sigma$ \\
Residual foregrounds & $2.12 \%$ & $0.09 \sigma$ \\
Total & $3.53 \%$ & $0.15 \sigma$ \\
\hline
\end{tabular}

Note. This is a list of systematic errors estimated for the lensing mass measurement.

foreground-free CMB map for the lensing reconstruction. This enhancement of foregrounds generally acts as an additional source of noise and tends to increase the variance of the reconstructed lensing maps. At the cluster locations, however, an increase in foreground emission due to galaxies inside the cluster can introduce undesired mode coupling between the estimated gradient map and the lensing map, resulting in a biased lensing signal. Since massive clusters host more galaxies, we can expect the bias to increase with the cluster mass or equivalently richness. Here we quantify this bias using the $\mathrm{S} 10$ foreground simulations. ${ }^{91}$

To this end, we begin with the simulated skies described in Section 3.2, to which we then add simulated clusters, including the lensing signal (only the 1-halo term), thermal and kinetic SZ effects, and emission associated with the cluster (e.g., from member galaxies). These simulations also include foregrounds uncorrelated with the clusters, such as field radio galaxies. The addition of foregrounds using S10 simulations is described as follows. Note that the foreground maps, whether associated with the cluster or not, are not lensed by the cluster in these simulations. The number of simulated clusters and their redshifts and richnesses are derived from the DES RM Year-3 fluxlimited sample. The richnesses and redshifts are converted to cluster masses according to the $M-\lambda$ relation (Equation (12)), with best-fit parameters from Melchior et al. (2017), $A_{M 17}=$ $2.35 \times 10^{14} M_{\odot}, \alpha_{M 17}=1.12$, and $\beta_{M 17}=0.18$.

For foregrounds, we extract half-arcminute resolution $300^{\prime} \times 300^{\prime}$ cutouts of the 95 and $150 \mathrm{GHz}$ S10 simulations of the tSZ, kSZ, radio, and infrared galaxies around halos corresponding to the mock cluster sample. We scale the tSZ power down from the S10 simulations by a factor of 1.75 to match the George et al. (2015) measurements. The S10 simulations contain $\sim 16,000$ halos above $M_{200 \mathrm{~m}} \geqslant 10^{14} M_{\odot}$ and 175 halos above $M_{200 \mathrm{~m}} \geqslant 5 \times 10^{14} M_{\odot}$ at redshifts $z \geqslant 0.25$. These foreground cutouts are added to our mock galaxy cluster-lensed CMB data sets. The maps are then processed in the same way as explained in Section 3.1, to extract the tSZ cleaned map, and passed into the QE.

We present the results in Figure 2. The true normalization is shown as the solid purple line. In the figure, the light shaded data points are the result for a single simulation run $(\sim 4000$ clusters) and the darker data points are the results for $10 \times$ the sample size. For our baseline analysis with SZ-free maps for

\footnotetext{
91 https://lambda.gsfc.nasa.gov/toolbox/tb_sim_ov.cfm
}

Simulations: Y3-flux limited: $\lambda \in[20,100]$

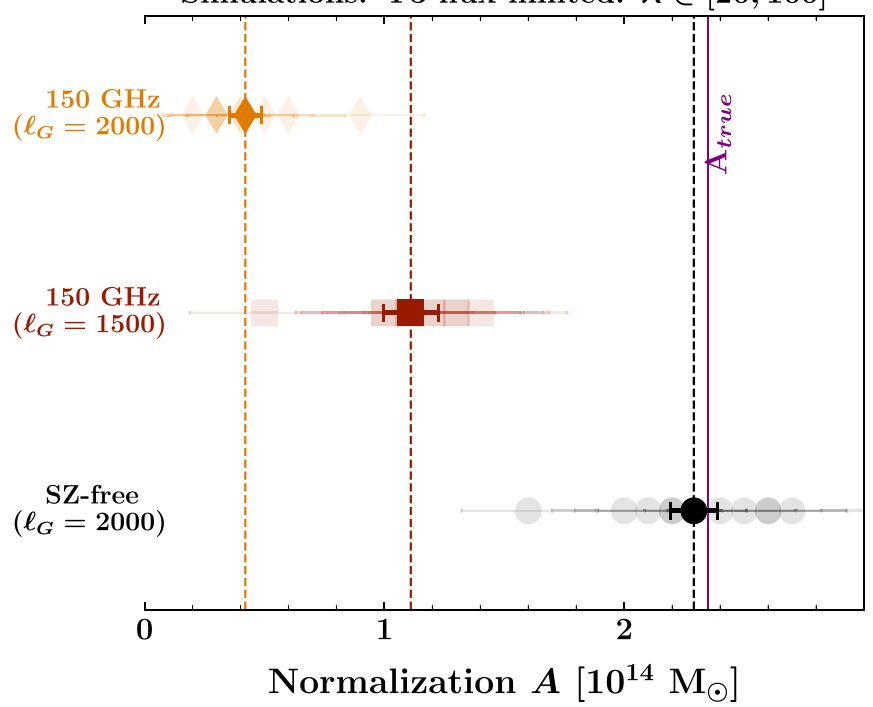

Figure 2. Quantifying the level of bias due to residual foregrounds and the tSZ signal using S10 simulations. The recovered lensing mass, unbiased for the fiducial case with SZ-free map for gradient estimation and $\ell_{G}=2000$, is shown as black circles. The equivalent biased results with just the $150 \mathrm{GHz}$ map and $\ell_{G}=1500$ (2000) cutoff scales for the gradient estimation are shown as red squares (orange diamonds). Each light shaded point corresponds to an individual simulation run with clusters from the DES RM Year-3 flux-limited sample. The darker data points are the values obtained for $10 \times$ the sample size.

the gradient estimation and $\ell_{G}=2000$, the recovered normalization is $\lesssim 0.5 \sigma$ from the true value. We obtain $A=2.30 \pm$ $0.09 \times 10^{14} M_{\odot}$ (black circle), implying no significant residual foreground bias in the lensing measurements. This result also provides evidence that the lensing pipeline is unbiased.

Comparison to standard QE: We also use the S10 simulations to compare the modified QE to the standard case using the $150 \mathrm{GHz}$ map for the background CMB gradient estimation. In the standard case, the correlations introduced between the two maps by the foregrounds, the tSZ signal in particular, can be alleviated by lowering the LPF threshold $\ell_{G}$ for the gradient map as in Equation (4). As described in Section 3.1, the choice of $\ell_{G}$ is a trade-off between the level of foreground bias and the lensing $\mathrm{S} / \mathrm{N}$. Here we adopt $\ell_{G}=2000$ and $\ell_{G}=1500$ and note that the results are heavily biased in both cases: red squares (orange diamonds) for $\ell_{G}=1500\left(\ell_{G}=2000\right)$. The level of bias is higher when $\ell_{G}$ is set to 2000 compared to 1500 , as expected. This bias is predominantly due to the tSZ signal and can be reduced by removing massive clusters from the analysis as in B18. For comparison, when we apply a richness cut of $\lambda \in[20,40]$, the lensing bias is reduced from $82 \%$ to $65 \%$ for $\ell_{G}=2000$ and $52 \%-35 \%$ for $\ell_{G}=1500$. This cut removes $\sim 500$ massive clusters from the analysis. This result can be compared to the conservative tSZ bias of $11 \%$ set by B 18 with $\ell_{G}=1500$ for the same richness range $\lambda \in[20,40]$. B18 obtained a lower bias value, as the high- $\ell$ modes in the SPT-SZ maps are downweighted due to $4 \times$ higher noise. This also suggests that we cannot handle the tSZ bias by simply removing clusters above a certain richness (e.g., $\lambda \gtrsim 40$ ) for low-noise CMB data sets.

Finally, a subtle point from the figure is that the mass constraints obtained using the $150 \mathrm{GHz}$ map for gradient estimation (orange diamonds) are better $(\sim 14 \%)$ than those obtained using the tSZ-free map for gradient estimation (black 
circles), despite adopting $\ell_{G}=2000$ in both cases. This hit in the $\mathrm{S} / \mathrm{N}$ arises because the SPTpol SZ-free map is noisier than the $150 \mathrm{GHz}$.

\subsubsection{Cluster Profile}

In our fiducial analysis, we assume that the underlying halo profile of the clusters follows the NFW DM model. However, in real clusters, deviations from the NFW profile have been observed (e.g., Diemer \& Kravtsov 2014), and Child et al. (2018) argued that the Einasto model is a better fit than NFW to stacked halo profiles.

In this section, we estimate the magnitude of a possible bias due to the assumption of the incorrect mass profile by using an Einasto profile (Einasto \& Haud 1989) to model the lensing convergence $\kappa^{1 h}$. The lensing $\mathrm{QE}$ and subsequently the reconstructed convergence maps remain unchanged. The Einasto profile is defined as

$$
\rho(r)_{\mathrm{Ein}}=\rho_{0} \exp \left(-\frac{2}{\alpha}\left[\left(\frac{r}{R_{\mathrm{S}}}\right)^{\alpha}-1\right]\right),
$$

where $\alpha=0.18$ is the shape parameter (Ludlow et al. 2013). As in the NFW analysis, the concentration $c_{200}$ as a function of mass and cluster redshift is obtained using the Duffy et al. (2008) relation. We use the general framework for spherically symmetric halos defined in R17, and simply plug the provided density profile into Equation (2.9) of R17 to obtain the Einasto convergence $\kappa^{1 h \text {,Ein }}$ profiles. The $\kappa^{2 h}$ term remains the same. For the Einasto case, we see a negligible shift of $0.01 \sigma$ compared to our fiducial result.

\subsubsection{Uncertainties in Filter Transfer Function and Beam}

As described in Section 2, the SPTpol map-making process is lossy, with noisy modes along the scan direction filtered out. The ideal, if computationally expensive, approach to handle the filtering would be an end-to-end simulation from the TOD to the lensing reconstruction. In this work, we take a computationally much cheaper approach and approximate the filtering by the phenomenological fit to the filter transfer function in Fourier space given by Equation (5). The major uncertainty is in the position of the high-pass filter (HPF) in the scan direction: this filters modes more strongly than the isotropic HPF, and the LPF is at angular scales that do not matter to the reconstruction. The estimated position for this HPF is $\ell_{x}=300 \pm 20$. We also recompute the models for an assumed $\ell_{x}=280$ and 320 to evaluate the shifts in the lensing masses. We note no significant effect (masses shift by roughly $\pm 0.02 \sigma$ ), indicating that the uncertainty in the simplified filtering treatment causes negligible changes to our results.

Similarly, we also check the effect of errors in the telescope beam modeling $B_{\ell}$ that were derived using Venus observations (see Section 2.1). We find that the effect due to beam uncertainties in the final result is also negligible. The shift in the lensing mass is $\lesssim 0.01 \sigma$ when we modify $B_{\ell} \rightarrow B_{\ell}+2 \sigma$.

\subsubsection{Uncertainties in $\mathrm{f}_{\text {mis }}$ Parameter}

In our baseline analysis, we perform a mis-centering correction of the cluster convergence models (see Section 3.6) using $f_{\text {mis }}=0.22$ based on the results by Rykoff et al. (2016) for the RM clusters from the science verification data. Now we generate new convergence models assuming a larger fraction, $33 \%$, of the clusters are mis-centered by modifying the miscentering parameter by its $1 \sigma$ error from Rykoff et al. (2016). Since the two parameters, $f_{\text {mis }}$ and $c_{\text {mis }}$, describing the cluster mis-centering are highly correlated, we also modify ln $c_{\text {mis }}=-1.32$ for this test.

The recovered mass increases by $2.8 \%$ in this case. However, the shift is only $0.12 \sigma, 1 / 8$ th of the statistical uncertainty. The direction of the shift is consistent with expectations, as assuming a larger $f_{\text {mis }}$ should smear the convergence model more than the fiducial case, leading to an increased lensing mass. Since this is the dominant systematic, we also estimate the error for the flux-limited sample. The mean lensing mass of the volume-limited sample goes up 3.2\%, but the change is still smaller than the statistical error $(0.16 \sigma)$.

\subsubsection{Underlying Cosmology}

The systematic error arising due to the assumption of a background cosmology is quantified here. As described in earlier sections, in our fiducial analysis we use the $\Lambda \mathrm{CDM}$ cosmology obtained using the Planck 2015 data sets (Planck Collaboration et al. 2016b). Here we repeat the analysis by modifying the lensed $\mathrm{CMB}$ power spectra $C_{\ell}$ to include the $1 \sigma$ errors to the Planck 2015 cosmological parameters. Modifying the background cosmology alters the weights of Equation (4) in the lensing estimator and also the model convergence profiles $\kappa^{1 h}(M, z)$ and $\kappa^{2 h}(M, z)$. However, the effect due to background cosmology in the inferred lensing mass is negligible with a shift in the lensing mass $\lesssim 0.03 \sigma$.

\section{Results and Discussion}

The main results of this work are the lensing-derived cluster mass constraints for the DES RM Year-3 cluster samples using SPTpol tSZ-free $\times 150 \mathrm{GHz}$ temperature maps. Below, we first present the lensing mass estimates in Section 5.1 and use the lensing measurements from the DES Year-3 volume-limited sample to independently calibrate the $M-\lambda$ relation of the cluster sample in Section 5.2. Finally, in Section 5.3 we compare our results to literature.

\subsection{Stacked Mass Measurements}

In Figure 3, we present the results of our stacked lensing measurements. The left (right) panel corresponds to the convergence maps stacked at the location of clusters in the DES Year-3 flux (volume)-limited sample. The variance in the flux-limited sample is lower than the volume-limited sample because the fluxlimited sample has twice as many objects. An estimate of the mean field has been subtracted from the maps. We reject the null hypothesis of no lensing with a significance of $8.7 \sigma$ for the fluxlimited sample of 4003 clusters. The obtained $\mathrm{S} / \mathrm{N}$ is consistent with our expectations from the simulations, shown as lighter black circles in Figure 2. For the smaller volume-limited sample, the nolensing hypothesis is ruled out at $6.7 \sigma$.

The radially binned convergence profiles that are used to estimate the cluster masses are shown in Figure 4, along with the best-fit model curves. The PTE values for the best-fit convergence models are 0.68 and 0.65 for the full- and volumelimited samples, respectively. The ringing pattern is because of the sharp filtering of modes above the SPTpol beam scale. The 

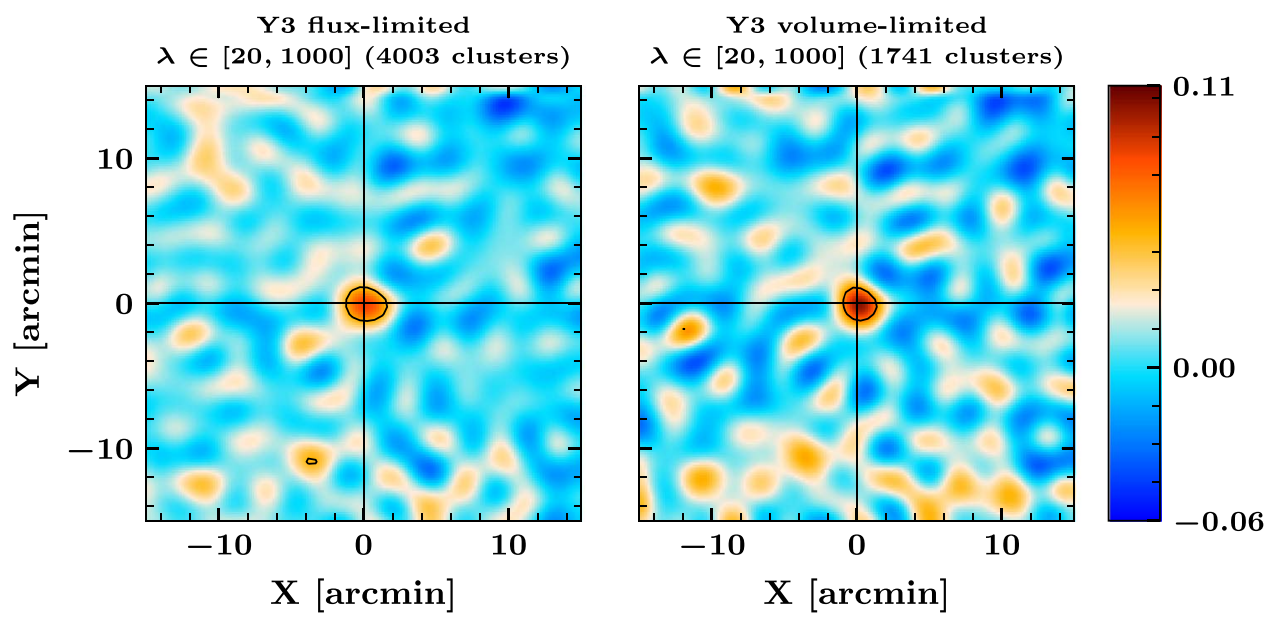

Figure 3. Inverse-variance weighted stacked convergence maps at the location of 4003 and 1741 clusters in the range $\lambda \in[20,1000]$ from the flux-limited (left) and the volume-limited (right) samples of the DES RM Year-3 cluster catalog. The contour corresponds to the regions above $3.5 \sigma$. The null hypothesis of no lensing is rejected at $8.7 \sigma$ and $6.7 \sigma$ for the two cases, respectively.

error bars plotted are the square root of the diagonal entries of the covariance matrix estimated using Equation (10). As explained in Section 3.6, all the mass estimates are derived by fitting a NFW profile along with the contribution from the 2-halo term to the measured radially binned profile. The recovered lensing masses for the stacked flux and volumelimited samples are $M_{200 \mathrm{~m}}=1.28_{-0.18}^{+0.14} \times 10^{14} M_{\odot}$ and $1.62_{-0.25}^{+0.32} \times 10^{14} M_{\odot}$, respectively. According to expectations, the lensing masses shift up by $0.3 \sigma$ when the 2-halo term is excluded.

A higher mean mass is expected for the volume-limited sample. At redshifts above $z \sim 0.6$, galaxies at the luminosity threshold adopted by RM become too faint to be detected in the DES data. Consequently, the richness of the clusters is extrapolated from the subset of galaxies that are sufficiently bright to be detected. This extrapolation introduces additional noise in the richness estimates. The increased scatter leads to more low-mass systems scattering up to apparently rich systems, thereby lowering the mean mass of the selected halos. For this reason, we restrict our analysis to the volumelimited sample in the subsequent sections.

\subsubsection{Comparison to B18 Analysis}

Now we discuss the differences in the analysis choices between B18 and this work to compare the lensing $\mathrm{S} / \mathrm{N}, 8.1 \sigma$ versus $6.7 \sigma$, obtained in the two works. $^{92}$ B18 used the $2500 \mathrm{deg}^{2}$ SPT-SZ data and DES RM Year-1 volume-limited sample in range $\lambda \in[20,40]$ with $2 \times$ more $^{93}$ clusters than this work. Here we use the SPTpol $150 \mathrm{GHz}$ map, which is $\sim 4 \times$ deeper than the SPT-SZ survey. However, as shown in Figure 2 of R17, the presence of foregrounds sets a floor to the achieved performance at low noise levels, and we note that the improvement in $\mathrm{S} / \mathrm{N}$ does not follow a simple scaling based on noise level.

\footnotetext{
92 We perform the comparison with the volume-limited sample, as B18 also performed the analysis with the DES Year-1 volume-limited sample.

93 Despite $5 \times$ larger sky coverage, the B18 cluster sample was only $2 \times$ larger than the one used here, because the overlap between the SPT-SZ survey and DES Year-1 data was only $40 \%$, compared to a nearly full overlap between DES 3-year data and the SPTpol survey.
}

\subsection{Mass-richness $M-\lambda$ Scaling Relation Calibration}

We now apply the lensing mass measurements from Section 5.1 to constrain the relationship between a cluster's mass, $M$, and optical richness, $\lambda$, in the DES RM Year-3 volume-limited sample. We limit the analysis to just the volume-limited sample because the flux-limited sample has selection bias, as explained in Section 5.1. Following earlier weak-lensing analyses of RM clusters (Melchior et al. 2017; McClintock et al. 2019; Simet et al. 2018), we use a power-law scaling relation for cluster mass, $M$, as a function of richness, $\lambda$, and redshift, $z$,

$$
M=A\left(\frac{\lambda}{40}\right)^{\alpha}\left(\frac{1+z}{1+0.35}\right)^{\beta},
$$

where $A$ is a normalization parameter, and the exponents $\alpha$ and $\beta$ are richness and redshift evolution parameters, respectively. The pivot points for the richness and redshift evolution were set based on DES weak-lensing measurements of M19. The model for the stacked mass is

$$
M(A, \alpha, \beta) \equiv M=\frac{\sum_{j} w_{j} M\left(\lambda_{j}, z_{j}\right)}{\sum_{j} w_{j}},
$$

where the sum runs over the number of clusters in the sample and the weight $w$ for each cluster is given in Equation (8).

We do not split the stacks into different richness or redshift bins. As a result, the data's sensitivity to the two evolution parameters is minimal, and we apply informative priors to both. We perform a Markov chain Monte Carlo analysis using the publicly available emcee (Foreman-Mackey et al. 2013) code to sample the likelihood space. We assume a flat prior for the normalization parameter $A$ in the range $A \in[0.1,100] \times$ $10^{14} M_{\odot}$. For the slopes, we use a Gaussian prior based on M19. Specifically, we set $\left(\alpha_{0}, \sigma_{\alpha}\right)=(1.356,0.056)$ and $\left(\beta_{0}, \sigma_{\beta}\right)=(-0.3,0.3)$. The posteriors on both the richness evolution parameters, as expected, follow the assumed prior. We obtain a normalization value of $A=2.70_{-0.50}^{+0.51} \times 10^{14} M_{\odot}$, which is consistent with results from DES weak-lensing measurements of $A_{\mathrm{DES}}=3.08 \pm 0.21 \times 10^{14} M_{\odot}$ by M19. The marginalized posterior for $A$ is shown as the black curve in 


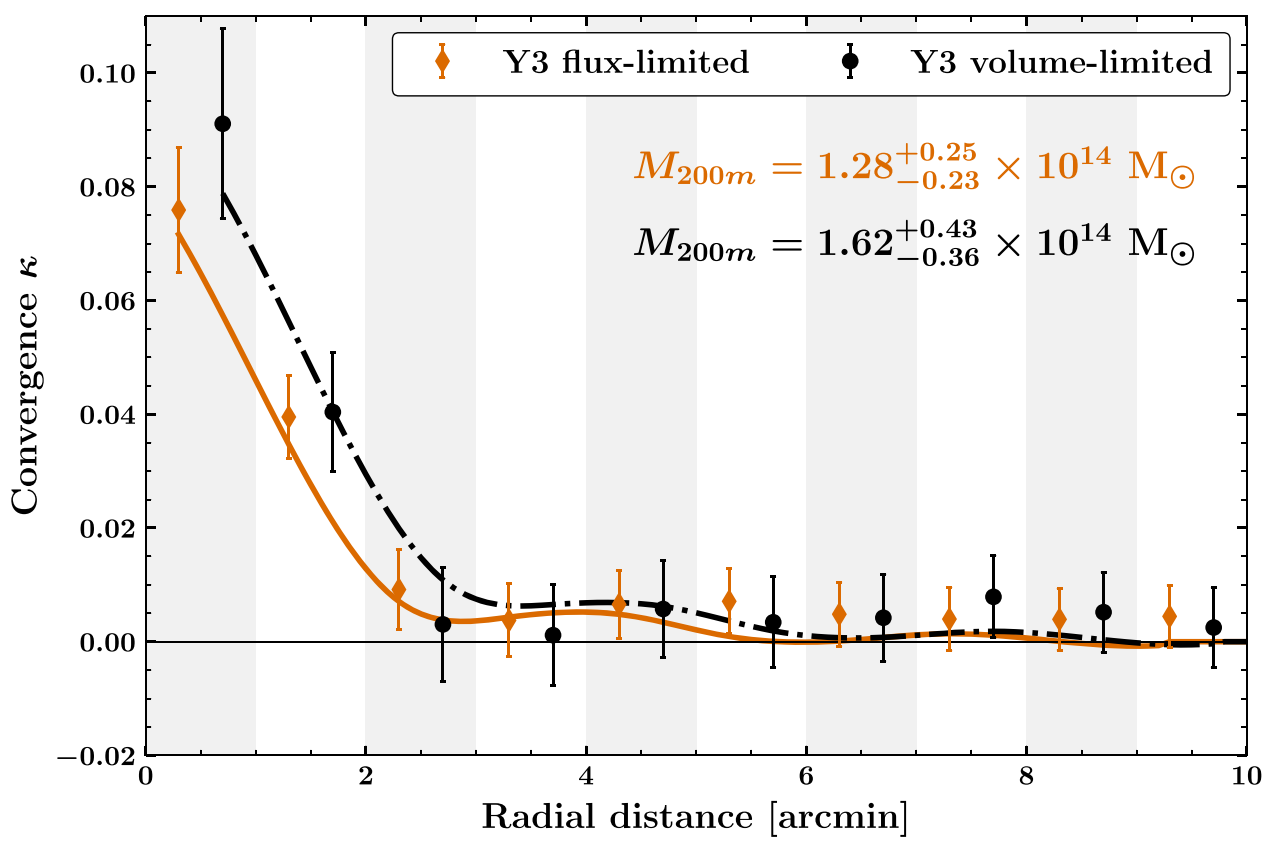

Figure 4. Azimuthally averaged radial profile of the stacked convergence maps from Figure 3. The black circles and orange diamonds correspond to the flux and volume-limited Year-3 DES RM cluster samples. The error bars are the diagonal value of the covariance matrix estimated using the jackknife technique in Equation (10). The data points for the two samples have been artificially shifted from the bin centers to avoid cluttering.

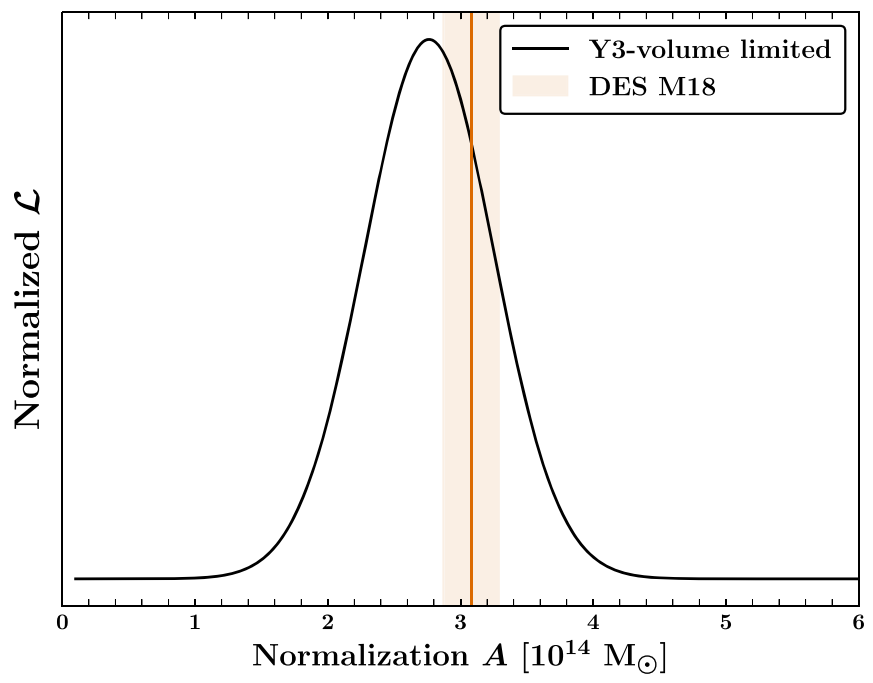

Figure 5. Marginalized posteriors of the normalization parameter $A$ of the $M-\lambda$ relation for the volume-limited sample of the RM cluster catalog. The result is consistent with the best-fit values obtained by DES weak-lensing measurements (M19), shown as the shaded region.

Figure 5, along with measurements from M19 as the orange shaded region.

\subsection{Comparison to Literature}

We now compare our results to similar works from the literature performed with the RM cluster catalogs from the SDSS and DES experiments. Since the richness estimated for a given cluster from surveys A and B can vary slightly, depending on the adopted data reduction and analysis choices, we include a small correction factor $\epsilon_{\mathrm{A}-\mathrm{B}}$ when comparing results from two surveys. We compute the ratio $\lambda_{\mathrm{A}} / \lambda_{\mathrm{B}}$ for the overlapping clusters in the two surveys and simply set $\epsilon_{\mathrm{A}-\mathrm{B}}$ to the median value of the ratios. We find the richness estimates in

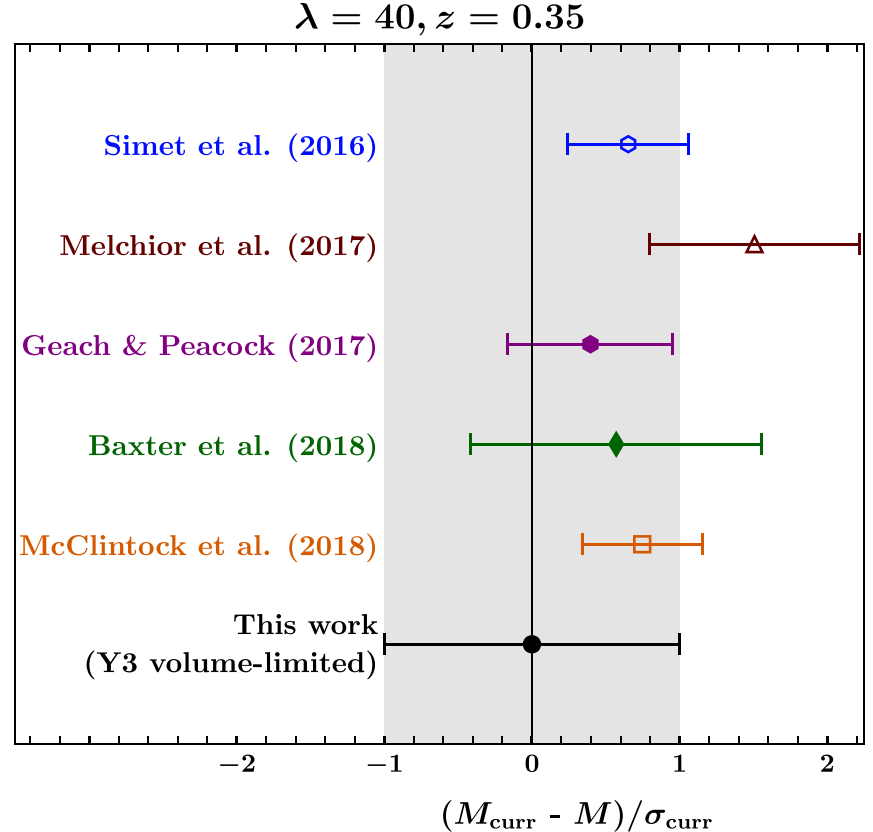

Figure 6. Comparison of $M_{200 \mathrm{~m}}$ mass estimates of galaxy clusters obtained using the $M-\lambda$ relation from different works in the literature using the RM cluster catalogs. The points have been normalized using the $1 \sigma$ error from the analysis with the Year-3 volume-limited sample of the DES RM catalog. The filled (open) points represent measurements using the CMB-cluster lensing (galaxy weak-lensing) technique.

DES Year-3 and Year-1 to be consistent with $\epsilon_{\mathrm{Y} 3-\mathrm{Y} 1}=1$. For the rest, we set $\epsilon_{\mathrm{Y} 3-\mathrm{SV}}=1.08$ and $\epsilon_{\mathrm{Y} 3-\mathrm{SDSS}}=0.93$ (M19). The comparison after including this correction factor is presented in Figure 6, which is similar to Figure 15 in M19. Specifically, we show the difference in $M_{200 \mathrm{~m}}$ masses obtained from different works for a cluster with richness $\lambda=40$ at redshift $z=0.35$, the pivot points in Equation (12). The figure is normalized 
using the $1 \sigma$ error from the current work with the Year-3 volume-limited DES RM catalog sample.

Each analysis uses a different cluster sample and lensing data. Simet et al. (2018) and Geach \& Peacock (2017) use the SDSS RM catalog sample containing roughly 26,000 clusters. Melchior et al. (2017) use the full catalog from the DES science verification data, while B18 and M19 perform the analysis using the DES Year-1 volume-limited sample. The works by Geach \& Peacock (2017) and B18 use the CMB-cluster lensing technique (filled points) with Planck and SPT-SZ CMB temperature maps. All the others use galaxy weak-lensing measurements and are represented as open points. As evident from the figure, our results are consistent with other similar works in the literature.

\section{Conclusions}

We have built a modified lensing QE to reconstruct lensing potential at the location of the DES RM clusters using the SPTpol $500 \mathrm{deg}^{2}$ field CMB temperature maps. We detect a stacked lensing signal at $8.7 \sigma$ and $6.7 \sigma$ level for the flux- and volume-limited samples of the Year-3 RM cluster catalog. The modified QE eliminates the tSZ-induced lensing bias by using two maps for lensing reconstruction: a low-noise SPTpol $150 \mathrm{GHz}$ map to reconstruct the small-scale lensing, and a tSZfree map to estimate the background CMB gradient. The tSZfree map is internal, constructed from the SPTpol 95 and $150 \mathrm{GHz}$ channels.

We model the lensing signal, assuming a NFW profile for the galaxy clusters, and find the stacked lensing masses to be $M_{200 \mathrm{~m}}=1.28_{-0.18}^{+0.14}$ [stat.] \pm 0.03 [sys.] and $1.62_{-0.25}^{+0.32}$ [stat.] \pm 0.04 [sys.] $\times 10^{14} M_{\odot}$ for the two catalog samples. The uncertainties in our knowledge about the cluster centroids are the dominant contributor $(\sim 3 \%)$ to the systematic error budget. We use the mass measurements from the volume-limited sample to calibrate the mass-richness $M-\lambda$ scaling relation of the RM galaxy clusters. The constraints on the richness and redshift evolution parameters are dominated by the priors assumed from the DES weak-lensing measurements M19. We obtain a best-fit normalization parameter of $A=2.70 \pm$ $0.51 \times 10^{14} M_{\odot}$. The results are consistent with other similar works in the literature, performed using the RM catalogs from DES and SDSS surveys. It must be noted that one must account for the Malmquist and Eddington biases (e.g., Allen et al. 2011) when using the provided scaling relation parameters to infer individual cluster masses for cosmological analysis with cluster abundance measurements. The Malmquist bias is due to selection effects, while the Eddington bias arises due to uncertainties in the inferred cluster masses, which tends to upscatter the low-mass clusters into higher mass bins. An an example, see the corrections performed by Battaglia et al. (2016) for the ACT and Penna-Lima et al. (2017) for the Planck cluster samples, respectively. However, these corrections are not required for the current analysis because we only measure the average mass of a set of clusters.

While CMB polarization data, which are almost unaffected by the presence of foregrounds, are expected to provide robust lensing estimates with the future low-noise $\mathrm{CMB}$ data sets like CMB-S4 R17, the estimator presented here and in a similar work by Madhavacheril \& Hill (2018) will be vital to extract lensing robustly from future low noise CMB temperature data sets.
We thank useful conversations with Mathew Madhavacheril at the Stanford CMB-lensing meeting in 2017, which partly inspired us in building this modified QE. The Melbourne group acknowledges support from the Australian Research Council's Discovery Projects scheme (DP150103208). S.R. also acknowledges partial support from the Laby Foundation. L.B.'s work was supported under the U.S. Department of Energy contract DE-AC02-06CH11357. We acknowledge the use of HEALPix (Górski et al. 2005) and CAMB (Lewis et al. 2000) routines.

This work was performed in the context of the South Pole Telescope scientific program. SPT is supported by the National Science Foundation through grant PLR-1248097. Partial support is also provided by the NSF Physics Frontier Center grant PHY-1125897 to the Kavli Institute of Cosmological Physics at the University of Chicago, the Kavli Foundation, and the Gordon and Betty Moore Foundation grant GBMF 947. This research used resources of the National Energy Research Scientific Computing Center (NERSC), a DOE Office of Science User Facility supported by the Office of Science of the U.S. Department of Energy under Contract No. DE-AC0205CH11231.

Funding for the DES Projects has been provided by the U.S. Department of Energy, the U.S. National Science Foundation, the Ministry of Science and Education of Spain, the Science and Technology Facilities Council of the United Kingdom, the Higher Education Funding Council for England, the National Center for Supercomputing Applications at the University of Illinois at Urbana-Champaign, the Kavli Institute of Cosmological Physics at the University of Chicago, the Center for Cosmology and Astro-Particle Physics at Ohio State University, the Mitchell Institute for Fundamental Physics and Astronomy at Texas A\&M University, Financiadora de Estudos e Projetos, Fundação Carlos Chagas Filho de Amparo à Pesquisa do Estado do Rio de Janeiro, Conselho Nacional de Desenvolvimento Científico e Tecnológico and the Ministério da Ciência, Tecnologia e Inovação, the Deutsche Forschungsgemeinschaft, and the Collaborating Institutions in the Dark Energy Survey.

The Collaborating Institutions are Argonne National Laboratory, the University of California at Santa Cruz, the University of Cambridge, Centro de Investigaciones Energéticas, Medioambientales y Tecnológicas-Madrid, the University of Chicago, University College London, the DES-Brazil Consortium, the University of Edinburgh, the Eidgenössische Technische Hochschule (ETH) Zürich, Fermi National Accelerator Laboratory, the University of Illinois at UrbanaChampaign, the Institut de Ciències de l'Espai (IEEC/CSIC), the Institut de Física d'Altes Energies, Lawrence Berkeley National Laboratory, the Ludwig-Maximilians Universität München and the associated Excellence Cluster Universe, the University of Michigan, the National Optical Astronomy Observatory, the University of Nottingham, Ohio State University, the University of Pennsylvania, the University of Portsmouth, SLAC National Accelerator Laboratory, Stanford University, the University of Sussex, Texas A\&M University, and the OzDES Membership Consortium.

The study is based in part on observations at Cerro Tololo Inter-American Observatory, National Optical Astronomy Observatory, which is operated by the Association of Universities for Research in Astronomy (AURA) under a cooperative agreement with the National Science Foundation. 
The DES data management system is supported by the National Science Foundation under grant No. AST-1138766 and AST-1536171. The DES participants from Spanish institutions are partially supported by MINECO under grants AYA2015-71825, ESP2015-66861, FPA2015-68048, SEV2016-0588, SEV-2016-0597, and MDM-2015-0509, some of which include ERDF funds from the European Union. IFAE is partially funded by the CERCA program of the Generalitat de Catalunya. Research leading to these results has received funding from the European Research Council under the European Union's Seventh Framework Program (FP7/20072013), including ERC grant agreements 240672, 291329, and 306478. We acknowledge support from the Australian Research Council Centre of Excellence for All-sky Astrophysics (CAASTRO), through project number CE110001020, and the Brazilian Instituto Nacional de Ciência e Tecnologia (INCT) e-Universe (CNPq grant 465376/2014-2).

This manuscript has been authored by Fermi Research Alliance, LLC, under Contract No. DE-AC02-07CH11359, with the U.S. Department of Energy, Office of Science, Office of High Energy Physics. The U.S. government retains-and the publisher, by accepting the article for publication, acknowledges that the U.S. government retains-a non-exclusive, paidup, irrevocable, worldwide license to publish or reproduce the published form of this manuscript, or allow others to do so, for US government purposes.

\section{ORCID iDs}

S. Raghunathan (1) https://orcid.org/0000-0003-1405-378X

E. Baxter (1) https://orcid.org/0000-0002-6836-3196

T. M. Crawford (1) https://orcid.org/0000-0001-9000-5013

C. L. Reichardt (10 https://orcid.org/0000-0003-2226-9169

P. A. R. Ade (1) https://orcid.org/0000-0002-5127-0401

J. Annis (1) https://orcid.org/0000-0002-0609-3987

F. Bianchini (i) https://orcid.org/0000-0003-4847-3483

J. P. Dietrich (ㄴ) https://orcid.org/0000-0002-8134-9591

J. García-Bellido (10 https://orcid.org/0000-0002-9370-8360

E. M. George (i) https://orcid.org/0000-0001-7874-0445

D. Gruen (1) https://orcid.org/0000-0003-3270-7644

R. A. Gruendl (1) https://orcid.org/0000-0002-4588-6517

T. de Haan (ํ) https://orcid.org/0000-0001-5105-9473

D. L. Hollowood (1) https://orcid.org/0000-0002-9369-4157

D. J. James (1) https://orcid.org/0000-0001-5160-4486

M. Carrasco Kind (1) https://orcid.org/0000-0002-4802-3194

K. Kuehn (다 https://orcid.org/0000-0003-0120-0808

P. Melchior (1) https://orcid.org/0000-0002-8873-5065

R. Miquel (1) https://orcid.org/0000-0002-6610-4836

D. Rapetti $\odot$ https://orcid.org/0000-0003-2196-6675

A. K. Romer (1) https://orcid.org/0000-0002-9328-879X

B. R. Saliwanchik (1) https://orcid.org/0000-0002-5089-7472

M. Soares-Santos (i) https://orcid.org/0000-0001-6082-8529

F. Sobreira (1) https://orcid.org/0000-0002-7822-0658

G. Tarle (i) https://orcid.org/0000-0003-1704-0781

W. L. K. Wu (1) https://orcid.org/0000-0001-5411-6920

Y. Zhang (i) https://orcid.org/0000-0001-5969-4631

\section{References}

Allen, S. W., Evrard, A. E., \& Mantz, A. B. 2011, ARA\&A, 49, 409 Applegate, D. E., von der Linden, A., Kelly, P. L., et al. 2014, MNRAS, 439, 48 Arnaud, M., Pratt, G. W., Piffaretti, R., et al. 2010, A\&A, 517, A92
Austermann, J. E., Aird, K. A., Beall, J. A., et al. 2012, Proc. SPIE, 8452, $84521 \mathrm{E}$

Bartelmann, M. 1996, A\&A, 313, 697

Battaglia, N., Leauthaud, A., Miyatake, H., et al. 2016, JCAP, 2016, 013

Baxter, E. J., Keisler, R., Dodelson, S., et al. 2015, ApJ, 806, 247

Baxter, E. J., Raghunathan, S., Crawford, T. M., et al. 2018, MNRAS, 476, 2674

Benson, B. A., Ade, P. A. R., Ahmed, Z., et al. 2014, Proc. SPIE, 9153 , 91531P

Bleem, L. E., Stalder, B., Brodwin, M., et al. 2015, ApJS, 216, 20

Calabretta, M. R., \& Greisen, E. W. 2002, A\&A, 395, 1077

Carlstrom, J. E., Ade, P. A. R., Aird, K. A., et al. 2011, PASP, 123, 568

Child, H. L., Habib, S., Heitmann, K., et al. 2018, ApJ, 859, 55

CMB-S4 Collaboration, Abazajian, K. N., Adshead, P., et al. 2016, arXiv: 1610.02743

Cooray, A., \& Sheth, R. 2002, PhR, 372, 1

de Haan, T., Benson, B. A., Bleem, L. E., et al. 2016, ApJ, 832, 95

Diemer, B., \& Kravtsov, A. V. 2014, ApJ, 789, 1

Dodelson, S. 2004, PhRvD, 70, 023009

Drlica-Wagner, A., Sevilla-Noarbe, I., Rykoff, E. S., et al. 2018, ApJS, 235, 33

Duffy, A. R., Schaye, J., Kay, S. T., \& Dalla Vecchia, C. 2008, MNRAS, 390, L64

Einasto, J., \& Haud, U. 1989, A\&A, 223, 89

Flaugher, B., Diehl, H. T., Honscheid, K., et al. 2015, AJ, 150, 150

Foreman-Mackey, D., Hogg, D. W., Lang, D., \& Goodman, J. 2013, PASP, 125,306

Geach, J. E., \& Peacock, J. A. 2017, NatAs, 1, 795

George, E. M., Reichardt, C. L., Aird, K. A., et al. 2015, ApJ, 799, 177

Górski, K. M., Hivon, E., Banday, A. J., et al. 2005, ApJ, 622, 759

Hartlap, J., Simon, P., \& Schneider, P. 2007, A\&A, 464, 399

Hasselfield, M., Hilton, M., Marriage, T. A., et al. 2013, JCAP, 7, 8

Henderson, S. W., Allison, R., Austermann, J., et al. 2016, JLTP, 184, 772

Henning, J. W., Sayre, J. T., Reichardt, C. L., et al. 2018, ApJ, 852, 97

Hoekstra, H., Bartelmann, M., Dahle, H., et al. 2013, SSRv, 177, 75

Holder, G., \& Kosowsky, A. 2004, ApJ, 616, 8

Horowitz, B., Ferraro, S., \& Sherwin, B. D. 2017, arXiv:1710.10236

Hu, W., DeDeo, S., \& Vale, C. 2007, NJPh, 9, 441

Lewis, A., \& Challinor, A. 2006, PhR, 429, 1

Lewis, A., Challinor, A., \& Lasenby, A. 2000, ApJ, 538, 473

LSST Science Collaboration, Abell, P. A., Allison, J., et al. 2009 , arXiv:0912.0201

Ludlow, A. D., Navarro, J. F., Boylan-Kolchin, M., et al. 2013, MNRAS, 432, 1103

Madhavacheril, M., Sehgal, N., Allison, R., et al. 2015, PhRvL, 114, 151302 Madhavacheril, M. S., \& Hill, J. C. 2018, PhRvD, 98, 023534

Mantz, A., Allen, S. W., Ebeling, H., \& Rapetti, D. 2008, MNRAS, 387, 1179

Mantz, A. B., von der Linden, A., Allen, S. W., et al. 2015, MNRAS, 446, 2205

Maturi, M., Bartelmann, M., Meneghetti, M., \& Moscardini, L. 2005, A\&A, 436, 37

McClintock, T., Varga, T. N., Gruen, D., et al. 2019, MNRAS, 482, 1352

Melchior, P., Gruen, D., McClintock, T., et al. 2017, MNRAS, 469, 4899

Melin, J.-B., \& Bartlett, J. G. 2015, A\&A, 578, A21

Merloni, A., Predehl, P., Becker, W., et al. 2012, arXiv:1209.3114

Morganson, E., Gruendl, R. A., Menanteau, F., et al. 2018, PASP, 130, 074501

Navarro, J. F., Frenk, C. S., \& White, S. D. M. 1996, ApJ, 462, 563

Oguri, M., \& Hamana, T. 2011, MNRAS, 414, 1851

Oguri, M., \& Takada, M. 2011, PhRvD, 83, 023008

Padin, S., Staniszewski, Z., Keisler, R., et al. 2008, ApOpt, 47, 4418

Penna-Lima, M., Bartlett, J. G., Rozo, E., et al. 2017, A\&A, 604, A89

Planck Collaboration, Ade, P. A. R., Aghanim, N., et al. 2016a, A\&A, 594, A24

Planck Collaboration, Ade, P. A. R., Aghanim, N., et al. 2016b, A\&A, 594, A13

Raghunathan, S., Bianchini, F., \& Reichardt, C. L. 2018, PhRvD, 98, 043506 Raghunathan, S., Patil, S., Baxter, E. J., et al. 2017, JCAP, 8, 030

Rozo, E., Rykoff, E. S., Abate, A., et al. 2016, MNRAS, 461, 1431

Rozo, E., Rykoff, E. S., Bartlett, J. G., \& Evrard, A. E. 2013, arXiv:1302.5086

Rozo, E., Wechsler, R. H., Rykoff, E. S., et al. 2010, ApJ, 708, 645

Rykoff, E. S., Rozo, E., Busha, M. T., et al. 2014, ApJ, 785, 104

Rykoff, E. S., Rozo, E., Hollowood, D., et al. 2016, ApJS, 224,

Salvati, L., Douspis, M., \& Aghanim, N. 2018, A\&A, 614, A13

Schaffer, K. K., Crawford, T. M., Aird, K. A., et al. 2011, ApJ, 743, 90

Sehgal, N., Bode, P., Das, S., et al. 2010, ApJ, 709, 920

Seljak, U. 2000, MNRAS, 318, 203

Seljak, U., \& Zaldarriaga, M. 2000, ApJ, 538, 57 
Simet, M., McClintock, T., Mandelbaum, R., et al. 2018, MNRAS, 480, 5385 Sunyaev, R. A., \& Zel'dovich, Y. B. 1972, CoASP, 4, 173

Sunyaev, R. A., \& Zeldovich, Y. B. 1980, MNRAS, 190, 413

The Simons Observatory Collaboration, Ade, P., Aguirre, J., et al. 2018, arXiv: 1808.07445

Tinker, J. L., Robertson, B. E., Kravtsov, A. V., et al. 2010, ApJ, 724, 878
Vikhlinin, A., Burenin, R. A., Ebeling, H., et al. 2009a, ApJ, 692, 1033

Vikhlinin, A., Kravtsov, A. V., Burenin, R. A., et al. 2009b, ApJ, 692, 1060 von der Linden, A., Allen, M. T., Applegate, D. E., et al. 2014, MNRAS, 439,2

Yoo, J., \& Zaldarriaga, M. 2008, PhRvD, 78, 083002

Yoo, J., Zaldarriaga, M., \& Hernquist, L. 2010, PhRvD, 81, 123006 